\title{
MULTIPLE BLOW-UP PHENOMENA FOR THE SINH-POISSON EQUATION
}

\author{
MASSIMO GROSSI AND ANGELA PISTOIA
}

AbstraCt. We consider the sinh-Poisson equation

$$
(P)_{\lambda} \quad-\Delta u=\lambda \sinh u \text { in } \Omega, u=0 \text { on } \partial \Omega,
$$

where $\Omega$ is a smooth bounded domain in $\mathbb{R}^{2}$ and $\lambda$ is a small positive parameter.

If $0 \in \Omega$ and $\Omega$ is symmetric with respect to the origin, for any integer $k$ if $\lambda$ is small enough, we construct a family of solutions to $(P)_{\lambda}$ which blows-up at the origin whose positive mass is $4 \pi k(k-1)$ and negative mass is $4 \pi k(k+1)$.

It gives a complete answer to an open problem formulated by Jost-Wang-Ye-Zhou in [Calc. Var. PDE (2008) 31: 263-276].

\section{INTRODUCTION}

In this paper we will study the semilinear elliptic equation

$$
-\Delta u=\lambda\left(e^{u}-e^{-u}\right) \quad \text { in } \Omega, \quad u=0 \quad \text { on } \partial \Omega,
$$

where $\Omega$ is a smooth bounded domain in $\mathbb{R}^{2}$ and $\lambda$ is a small positive parameter.

This problem arises in plasma physics and statistical mechanics. See, for instance, Chorin [5. Marchioro-Pulvirenti [21] and the references therein.

This problem also plays a very important role in the study of the construction of constant mean curvature surfaces initiated by Wente [30, 31.

In 1988 Spruck 28 studied (1.1) when $\Omega$ contains the origin and it is a domain symmetric with respect to reflections about the $x_{1}$ and $x_{2}$ axes. In particular, he proved that a sequence of nontrivial solutions $u_{n}$ of (1.1) with $\lambda_{n} \rightarrow 0$ is such that $u_{n}(x) \rightarrow-2 \ln |g(x)|^{2}$ uniformly on compact subsets of $\Omega \backslash\{0\}$, where $g$ is the symmetric conformal map of $\Omega$ onto the unit disk. Twenty years later Jost-Wang-Ye-Zhou [19] investigated the blow-up analysis of solutions to (1.1) and they give a more precise asymptotic behavior when the sequence of solutions $u_{n}$ blows-up as $\lambda_{n} \rightarrow 0$. Let us define the positive and the negative blow-up set of the sequence $u_{n}$ respectively by

$$
\begin{aligned}
& S_{+}:=\left\{x \in \Omega: \exists x_{n} \rightarrow x \text { s.t. } u_{n}\left(x_{n}\right) \rightarrow+\infty\right\} \\
& S_{-}:=\left\{x \in \Omega: \exists x_{n} \rightarrow x \text { s.t. } u_{n}\left(x_{n}\right) \rightarrow-\infty\right\} .
\end{aligned}
$$

For any $x_{0} \in S_{+} \cup S_{-}$let us define the positive and the negative mass of $x_{0}$ respectively by

$$
m_{+}\left(x_{0}\right):=\lim _{r \rightarrow 0} \lim _{n} \int_{B\left(x_{0}, r\right)} \lambda_{n} e^{u_{n}(x)} d x, m_{-}\left(x_{0}\right):=\lim _{r \rightarrow 0} \lim _{n} \int_{B\left(x_{0}, r\right)} \lambda_{n} e^{-u_{n}(x)} d x .
$$

Jost-Wang-Ye-Zhou [19] proved that $S_{+}$and $S_{-}$are finite sets and that the masses $m_{+}\left(x_{0}\right)$ and $m_{-}\left(x_{0}\right)$ are multiple of $8 \pi$. This is an analogue of the result of Li-Shafrir for the Gelfand problem

$$
-\Delta u=\lambda e^{u} \quad \text { in } \Omega, \quad u=0 \quad \text { on } \partial \Omega .
$$

In view of the relationship established by Ohtsuka-Suzuki 25

$$
\left(m_{+}\left(x_{0}\right)-m_{-}\left(x_{0}\right)\right)^{2}=8 \pi\left(m_{+}\left(x_{0}\right)+m_{-}\left(x_{0}\right)\right)
$$

it follows that for any $x_{0} \in S_{+} \cup S_{-}$

$$
m_{+}\left(x_{0}\right)=4 \pi k(k-1) \text { and } m_{-}\left(x_{0}\right)=4 \pi k(k+1)
$$

Date: August 4, 2018.

1991 Mathematics Subject Classification. 35J60, 35B33, 35J25, 35J20, 35B40.

Key words and phrases. Blow up values, tower of bubbles, singular Liouville problems. 
or

$$
m_{+}\left(x_{0}\right)=4 \pi k(k+1) \text { and } m_{-}\left(x_{0}\right)=4 \pi k(k-1)
$$

for some integers $k \geq 1$. When $k=1$ we say that $x_{0}$ is a simple (positive or negative) blow-up point, while if $k \geq 2$ we say that $x_{0}$ is a multiple (nodal) blow-up point.

Bartolucci-Pistoia [1] and Bartsch-Pistoia-Weth 3] constructed sign-changing solutions to (1.1) with one or more simple positive and simple negative blow-up points. The solutions they found are sum of standard bubbles which solve the Liouville problem

$$
-\Delta w=e^{w} \quad \text { in } \quad \mathbb{R}^{2}, \quad \int_{\mathbb{R}^{2}} e^{w(x)} d x<+\infty .
$$

As far as it concerns existence of solutions with multiple blow-up points, in 19 the authors asked the following question.

(Q) Is it possible to find solutions to problem (1.1) with a multiple nodal blow-up point, i.e. $k \geq 2$.

In this paper we give a positive answer to this question. The result we have is

Theorem 1.1. Assume $0 \in \Omega$ and $\Omega$ is symmetric with respect to the origin, i.e. $x \in \Omega$ iff $-x \in \Omega$.

For any integer $k$, there exists $\lambda_{k}>0$ such that for any $\lambda \in\left(0, \lambda_{k}\right)$ problem (1.1) has a sign-changing solution $u_{\lambda}$ such that $u_{\lambda}(x)=u_{\lambda}(-x)$ and

$$
u_{\lambda}(x) \rightarrow(-1)^{k} 8 \pi k G(x, 0) \text { uniformly on compact subsets of } \Omega \backslash\{0\} \text { as } \lambda \rightarrow 0 .
$$

Moreover, the origin is a multiple nodal blow-up point whose blow up values are

$$
m_{-}(0)=4 \pi k(k+1) \text { and } m_{+}(0)=4 \pi k(k-1) \text { if } k \text { is even }
$$

and

$$
m_{-}(0)=4 \pi k(k-1) \text { and } m_{+}(0)=4 \pi k(k+1) \text { if } k \text { is odd. }
$$

Here

$$
G(x, y)=\frac{1}{2 \pi} \ln \frac{1}{|x-y|}+H(x, y), \quad x, y \in \Omega
$$

is the Green's function of the Dirichlet Laplacian in $\Omega$ and $H(x, y)$ is its regular part.

The solution $u_{\lambda}$ is constructed by superposing $k$ different kind of bubbles with alternating sign. Each bubble solves a different singular Liouville problem

$$
-\Delta w=|x|^{\alpha-2} e^{w} \quad \text { in } \quad \mathbb{R}^{2}, \quad \int_{\mathbb{R}^{2}}|x|^{\alpha-2} e^{w(x)} d x<+\infty
$$

for a suitable choice of $k$ different $\alpha$ 's (see (2.4) $)$. The choice of $\alpha$ 's is a crucial point in the construction of the solution. We will show in Section 2 that necessarily

$$
\alpha_{i}=4 i-2 \text { for any } i=1, \ldots, k \text {. }
$$

We remark that when $\alpha=2$ problem (1.8) reduces to the well known Liouville equation (1.3) whose solutions have been classified by Chen-Li 4 to be radially symmetric. When $\alpha>2$ is an integer all solutions to (1.8) have been classified by Prajapat-Tarantello [27. In this case problem (1.8) has radial and non-radial solutions. Our construction just relies on the radial ones.

Even if the solution we find resembles a tower of bubbles, it is important to point out that it is a new kind of tower of bubbles. Indeed, classical tower of bubbles are constructed by superposing bubbles which solve the same limit problem in the whole space, while our solution is constructed by superposing different bubbles which are solutions to different limit problems in $\mathbb{R}^{2}$.

This is a new phenomena: the solution we find is generated by cooking up bubbles related to different limit problems. The existence of this new kind of solutions was suggested by a recent result due to Grossi-Grumiau-Pacella [18. They study the asymptotic behavior of the least energy nodal radial solution to the problem

$$
-\Delta u=|u|^{p-1} u \quad \text { in } B, \quad u=0 \quad \text { on } \partial B
$$


where $B$ is the unit ball in $\mathbb{R}^{2}$ and the exponent $p$ goes to $+\infty$. In particular, they prove that the positive and the negative parts of this solution (suitable scaled) converge to the solutions of the limit problems (1.8) with two different values of $\alpha$ 's.

We recall that classical towers of bubbles were constructed for some critical problems in $\mathbb{R}^{n}$ with $n \geq 3$. In particular, towers of positive bubbles were found by Del Pino-DolbeaultMusso [6, 7, Ge-Jing-Pacard [15] and Del Pino-Musso-Pistoia [12, while towers of signchanging bubbles were built by Pistoia-Weth 26, Musso-Pistoia 23, 24, and Ge-MussoPistoia [16]. See also Esposito-Wei [14] for a related problem with Neumann boundary condition and Del Pino-Dolbeault-Musso 8 for a problem with the $p$-Laplacian operator.

We want to emphasize that in the present paper the idea of using bubbles related to different limit problems is crucial! Indeed, the proof could not work if we argue as in all the previous papers, where the same bubbles always is used to build the solution.

We also want to point out that an extremely delicate point in the paper concerns the linear theory developed in Section 4 . In this framework some new ideas are necessary. Moreover, we remark that our approach also simplifies the linear theory studied in [13] and [11.

Finally, we believe that Theorem 1.1 holds even if we drop the assumption on the symmetry of $\Omega$. More precisely, we conjecture that in any domain $\Omega$ it is possible to construct a family of sign-changing solutions which blows-up at the maximum point of the Robin's function with the prescribed blow-up values.

The proof of our result relies on a contraction mapping argument. The paper is organized as follows. In Section 2 we establish some preliminary estimates. In Section 3 we estimate the error term. In Section 4 we study a linear problem. In Section 5 we complete the proof of Theorem 1.1] In Appendix we write some useful facts.

Acknowledgments. The authors would like to thank F. Pacella for many helpful discussions.

\section{The ANSATZ And The CHOICE OF $\alpha$ 's}

Let $\alpha \geq 2$. Let us introduce the functions

$$
w_{\delta}^{\alpha}(x):=\ln 2 \alpha^{2} \frac{\delta^{\alpha}}{\left(\delta^{\alpha}+|x|^{\alpha}\right)^{2}} \quad x \in \mathbb{R}^{2}, \delta>0
$$

which solve the problem (1.8).

Let us introduce the projection $P u$ of a function $u$ into $H_{0}^{1}(\Omega)$, i.e.

$$
\Delta P u=\Delta u \quad \text { in } \Omega, \quad P u=0 \quad \text { on } \partial \Omega .
$$

Let $k$ be a fixed integer. We look for a sign changing solution to (1.1) as

$$
u_{\lambda}(x):=W_{\lambda}(x)+\phi_{\lambda}(x), \quad W_{\lambda}(x):=\sum_{i=1}^{k}(-1)^{i} P w_{\delta_{i}}^{\alpha_{i}}(x)
$$

where for any $i=1, \ldots, k$ the $\alpha_{i}$ 's satisfy

$$
\alpha_{i}:=4 i-2
$$

and the concentration parameters satisfy

$$
\delta_{i}:=d_{i} \lambda^{\frac{2(k-i)+1}{\alpha_{i}}}=d_{i} \lambda^{\frac{2(k-i)+1}{4 i-2}} \text { for some } d_{i}>0 .
$$

It is important to point out that by (2.4) and (2.5) we deduce

$$
\frac{\delta_{i}}{\delta_{i+1}}=\frac{d_{i}}{d_{i+1}} \lambda^{\frac{2 k}{4 i^{2}-1}} \rightarrow 0 \text { as } \lambda \rightarrow 0 .
$$

The rest term $\phi_{\lambda}$ will be choose in the space $\mathrm{H}_{0}^{1}(\Omega)$ and will be symmetric with respect to the origin, i.e. $\phi(x)=\phi(-x)$ for any $x \in \Omega$.

The choice of $\delta_{i}$ 's and $\alpha_{i}$ 's is motivated by the need for the interaction among bubbles to be small. Indeed, an important feature is that each bubble interacts with all the other ones 
and in general the interaction is not negligible! The interaction will be measured in Lemma 3.1 using the function

$$
\begin{aligned}
\Theta_{j}(y) & :=(-1)^{j} W_{\lambda}\left(\delta_{j} y\right)-w_{j}\left(\delta_{j} y\right)-\left(\alpha_{j}-2\right) \ln \left|\delta_{j} y\right|+\ln \lambda \\
& =P w_{j}\left(\delta_{j} y\right)-w_{j}\left(\delta_{j} y\right)-\left(\alpha_{j}-2\right) \ln \left|\delta_{j} y\right|+\sum_{i \neq j}(-1)^{i-j} P w_{i}\left(\delta_{j} y\right)+\ln \lambda .
\end{aligned}
$$

The choice of parameters $\alpha_{j}$ and $\delta_{j}$ made in (2.8) and (2.9) (which imply (2.4) and (2.5)) ensures that $\Theta_{j}$ is small. Roughly speaking, the choice of $\alpha_{j}$ allows to kill the interaction among the $j$-th bubble and all the precedent (faster) bubbles, while the choice of $\delta_{j}$ allows to kill the interaction among the $j$-th bubble and all the consecutive (slower) bubbles. More precisely, in order to have $\Theta_{j}$ small in Lemma 2.2 we will need to choose $\delta_{j}$ 's and $\alpha_{j}$ 's so that

$$
\left(\alpha_{j}-2\right)+2 \sum_{\substack{i=1 \\ i<j}}^{k}(-1)^{i-j} \alpha_{i}=0
$$

and

$$
-\alpha_{j} \ln \delta_{j}-2 \sum_{\substack{i=1 \\ i>j}}^{k}(-1)^{i-j} \alpha_{i} \ln \delta_{i}-\ln \left(2 \alpha_{j}^{2}\right)+\sum_{i=1}^{k}(-1)^{i-j} h_{i}(0)+\ln \lambda=0,
$$

where we agree that if $j=1$ or $j=k$ the sum over the indices $i<j$ or $i>j$ is zero, respectively. Here $h_{i}(x):=4 \pi \alpha_{i} H(x, 0)$.

By (2.8) we immediately deduce that

$$
\alpha_{1}=2 \text { and } \alpha_{j+1}=\alpha_{j}+4 \text { for } j=2, \ldots, k-1,
$$

which implies (2.4) and by (2.9) we immediately deduce that

$$
\delta_{k}^{\alpha_{k}}=\frac{e^{-\sum_{i=1}^{k}(-1)^{i-k} h_{i}(0)}}{2 \alpha_{k}^{2}} \lambda
$$

and

$$
\delta_{j}^{\alpha_{j}}=\left(4 \alpha_{j}^{2} \alpha_{j+1}^{2} e^{-2 \sum_{i=1}^{k}(-1)^{i-j} h_{i}(0)}\right) \delta_{j+1}^{\alpha_{j+1}} \lambda^{2} \text { for } j=1, \ldots, k-1,
$$

which implies (2.5). We also remark that

$$
\sum_{i=1}^{k}(-1)^{i-j} h_{i}(0)=(-1)^{-j} 4 \pi H(0,0) \sum_{i=1}^{k}(-1)^{i} \alpha_{i}=(-1)^{k-j} 8 k \pi H(0,0),
$$

because by (2.4) we easily deduce

$$
\sum_{i=1}^{k}(-1)^{i} \alpha_{i}=(-1)^{k} 2 k
$$

In order to estimate $\Theta_{j}$ we need to introduce the following set of shrinking annulus.

For any $j=1, \ldots, k$ we set

$$
A_{j}:=\left\{x \in \Omega: \sqrt{\delta_{j-1} \delta_{j}} \leq|x| \leq \sqrt{\delta_{j} \delta_{j+1}}\right\}, j=1, \ldots, k
$$

where we set $\delta_{0}:=0$ and $\delta_{k+1}:=+\infty$.

We point out that if $j, \ell=1, \ldots, k$

$$
\frac{A_{j}}{\delta_{\ell}}=\left\{y \in \frac{\Omega}{\delta_{\ell}}: \frac{\sqrt{\delta_{j-1} \delta_{j}}}{\delta_{\ell}} \leq|y| \leq \frac{\sqrt{\delta_{j} \delta_{j+1}}}{\delta_{\ell}}\right\}
$$

and so roughly speaking $\frac{A_{j}}{\delta_{\ell}}$ shrinks to the origin if $\ell<j, \frac{A_{j}}{\delta_{j}}$ invades the whole space $\mathbb{R}^{2}$ and $\frac{A_{j}}{\delta_{\ell}}$ runs off to infinity if $\ell>j$. that

For sake of simplicity, we set $w_{i}:=w_{\delta_{i}}^{\alpha_{i}}(x)$. By the maximum principle we easily deduce 


\section{Lemma 2.1.}

$$
\begin{aligned}
P w_{i}(x) & =w_{i}(x)-\ln \left(2 \alpha_{i}^{2} \delta_{i}^{\alpha_{i}}\right)+h_{i}(x)+O\left(\delta_{i}^{\alpha_{i}}\right) \\
& =-2 \ln \left(\delta_{i}^{\alpha_{i}}+|x|^{\alpha_{i}}\right)+h_{i}(x)+O\left(\delta_{i}^{\alpha_{i}}\right)
\end{aligned}
$$

and for any $i, j=1, \ldots, k$

$$
P w_{i}\left(\delta_{j} y\right)=\left\{\begin{array}{cc}
-2 \alpha_{i} \ln \left(\delta_{j}|y|\right)+h_{i}(0) & \\
+O\left(\frac{1}{|y|^{\alpha_{i}}}\left(\frac{\delta_{i}}{\delta_{j}}\right)^{\alpha_{i}}\right)+O\left(\delta_{j}|y|\right)+O\left(\delta_{i}^{\alpha_{i}}\right) & \text { if } i<j, \\
-2 \alpha_{i} \ln \delta_{i}-2 \ln \left(1+|y|^{\alpha_{i}}\right)+h_{i}(0) & \text { if } i=j, \\
+O\left(\delta_{i}|y|\right)+O\left(\delta_{i}^{\alpha_{i}}\right) & \\
-2 \alpha_{i} \ln \delta_{i}+h_{i}(0) & \\
+O\left(|y|^{\alpha_{i}}\left(\frac{\delta_{j}}{\delta_{i}}\right)^{\alpha_{i}}\right)+O\left(\delta_{j}|y|\right)+O\left(\delta_{i}^{\alpha_{i}}\right) & \text { if } i>j .
\end{array}\right.
$$

Here $h_{i}(x):=4 \pi \alpha_{i} H(x, 0)$.

Now, we are in position to prove the following crucial estimates.

Lemma 2.2. Assume (2.8) and (2.9). For any $j=1, \ldots, k$ we have

and in particular

$$
\left|\Theta_{j}(y)\right|=O\left(\delta_{j}|y|+\lambda\right) \quad \text { for any } y \in \frac{A_{j}}{\delta_{j}}
$$

$$
\sup _{y \in \frac{A_{j}}{\delta_{j}}}\left|\Theta_{j}(y)\right|=O(1) .
$$

Proof. First of all, it is useful to estimate the projection $P w_{i}$.

By Lemma 2.1 (also using the mean value theorem $h_{j}\left(\delta_{j}|y|\right)=h_{j}(0)+O\left(\delta_{j}|y|\right)$ ), by (2.8) and by 2.9) we deduce

$$
\begin{aligned}
& \Theta_{j}(y)=\left[-\alpha_{j} \ln \delta_{j}-\ln \left(2 \alpha_{j}^{2}\right)+h_{j}(0)+O\left(\delta_{j}|y|\right)+O\left(\delta_{j}^{\alpha_{j}}\right)\right]-\left(\alpha_{j}-2\right) \ln \left|\delta_{j} y\right| \\
& +\sum_{i<j}(-1)^{i-j}\left[-2 \alpha_{i} \ln \left(\delta_{j}|y|\right)+h_{i}(0)+O\left(\frac{1}{|y|^{\alpha_{i}}}\left(\frac{\delta_{i}}{\delta_{j}}\right)^{\alpha_{i}}\right)+O\left(\delta_{j}|y|\right)+O\left(\delta_{i}^{\alpha_{i}}\right)\right] \\
& +\sum_{i>j}(-1)^{i-j}\left[-2 \alpha_{i} \ln \delta_{i}+h_{i}(0)+O\left(|y|^{\alpha_{i}}\left(\frac{\delta_{j}}{\delta_{i}}\right)^{\alpha_{i}}\right)+O\left(\delta_{j}|y|\right)+O\left(\delta_{i}^{\alpha_{i}}\right)\right] \\
& +\ln \lambda \\
& =\underbrace{\left[-\alpha_{j} \ln \delta_{j}-2 \sum_{i>j}(-1)^{i-j} \alpha_{i} \ln \delta_{i}-\ln \left(2 \alpha_{j}^{2}\right)+\sum_{i=1}^{k}(-1)^{i-j} h_{i}(0)+\ln \lambda\right]} \\
& =0 \text { because of 2.9 } \\
& -\underbrace{\left[\left(\alpha_{j}-2\right)+2 \sum_{i<j}(-1)^{i-j} \alpha_{i}\right]} \ln \left(\delta_{j}|y|\right) \\
& =0 \text { because of (2.8) } \\
& +O\left(\delta_{j}|y|\right)+\sum_{i=1}^{k} O\left(\delta_{i}^{\alpha_{i}}\right)+\sum_{i<j} O\left(\frac{1}{|y|^{\alpha_{i}}}\left(\frac{\delta_{i}}{\delta_{j}}\right)^{\alpha_{i}}\right)+\sum_{i>j} O\left(|y|^{\alpha_{i}}\left(\frac{\delta_{j}}{\delta_{i}}\right)^{\alpha_{i}}\right) \\
& =O\left(\delta_{j}|y|\right)+\sum_{i=1}^{k} O\left(\delta_{i}^{\alpha_{i}}\right)+\sum_{i<j} O\left(\frac{1}{|y|^{\alpha_{i}}}\left(\frac{\delta_{i}}{\delta_{j}}\right)^{\alpha_{i}}\right)+\sum_{i>j} O\left(|y|^{\alpha_{i}}\left(\frac{\delta_{j}}{\delta_{i}}\right)^{\alpha_{i}}\right) .
\end{aligned}
$$


By (2.5) we deduce that

$$
O\left(\delta_{i}^{\alpha_{i}}\right)=O\left(\lambda^{2 k-2 i+1}\right)=O(\lambda) \text { because } 1 \leq i \leq k
$$

Moreover, if $y \in \frac{A_{j}}{\delta_{j}}$ then $\sqrt{\frac{\delta_{j-1}}{\delta_{j}}} \leq|y| \leq \sqrt{\frac{\delta_{j+1}}{\delta_{j}}}$ and so if $j=2, \ldots, k$ and $i<j$ we have

$$
\begin{aligned}
O\left(\frac{1}{|y|^{\alpha_{i}}}\left(\frac{\delta_{i}}{\delta_{j}}\right)^{\alpha_{i}}\right) & =O\left(\left(\frac{\delta_{i}^{2}}{\delta_{j-1} \delta_{j}}\right)^{\frac{\alpha_{i}}{2}}\right)=O\left(\left(\frac{\delta_{j-1}}{\delta_{j}}\right)^{\frac{\alpha_{i}}{2}}\right)=O\left(\lambda^{\frac{2 k}{4(j-1)^{2}-1}(2 i-1)}\right) \\
& =O\left(\lambda^{\frac{2 k}{2 k-1}}\right)=O(\lambda),
\end{aligned}
$$

(since the minimum of $\lambda$ 's exponent is achieved when $i=j-1$ and $j=k$ ) and if $j=$ $1, \ldots, k-1$ and $i>j$ we have

$$
\begin{aligned}
O\left(|y|^{\alpha_{i}}\left(\frac{\delta_{j}}{\delta_{i}}\right)^{\alpha_{i}}\right) & =O\left(\left(\frac{\delta_{j+1} \delta_{j}}{\delta_{i}^{2}}\right)^{\frac{\alpha_{i}}{2}}\right)=O\left(\left(\frac{\delta_{j}}{\delta_{j+1}}\right)^{\frac{\alpha_{i}}{2}}\right)=O\left(\lambda^{\frac{2 k}{4 j^{2}-1}(2 i-1)}\right) \\
& =O\left(\lambda^{\frac{2 k}{2 k-3}}\right)=O(\lambda),
\end{aligned}
$$

(since the minimum of $\lambda$ 's exponent is achieved when $i=j+1$ and $j=k-1$ ). Collecting all the previous estimates, we get (2.18).

Estimate (2.19) follows immediately by (2.18), because if $y \in \frac{A_{j}}{\delta_{j}}$ then $\delta_{j}|y|=O(1)$.

In the following, we will denote by

$$
\|u\|_{p}:=\left(\int_{\Omega}|u(x)|^{p} d x\right)^{\frac{1}{p}} \text { and }\|u\|:=\left(\int_{\Omega}|\nabla u(x)|^{2} d x\right)^{\frac{1}{2}}
$$

the usual norms in the Banach spaces $\mathrm{L}^{p}(\Omega)$ and $\mathrm{H}_{0}^{1}(\Omega)$, respectively.

\section{Estimate OF THE ERROR TERM}

In this section we will estimate the two following error terms

$$
\begin{gathered}
\mathcal{R}_{\lambda}(x):=-\Delta W_{\lambda}(x)-\lambda f\left(W_{\lambda}(x)\right), x \in \Omega \\
\mathcal{S}_{\lambda}(x):=\lambda f^{\prime}\left(W_{\lambda}(x)\right)-\sum_{i=1}^{k} 2 \alpha_{i}^{2} \frac{|x|^{\alpha_{i}-2}}{\left(\delta_{i}^{\alpha_{i}}+|x|^{\alpha_{i}}\right)^{2}}, x \in \Omega .
\end{gathered}
$$

Here $f(s):=e^{s}-e^{-s}$.

Lemma 3.1. Let $\mathcal{R}_{\lambda}$ as in (3.1). There exists $\epsilon>0$ such that for any $p \in[1,1+\epsilon)$ we have

$$
\left\|\mathcal{R}_{\lambda}\right\|_{p}=O\left(\lambda^{\frac{2-p}{2 p(2 k-1)}}\right)
$$

Proof. First of all we observe that

$$
\mathcal{R}_{\lambda}(x)=\sum_{i=1}^{k}(-1)^{i}|x|^{\alpha_{i}-2} e^{w_{i}(x)}-\lambda e^{\sum_{i=1}^{k}(-1)^{i} P w_{i}(x)}+\lambda e^{\sum_{i=1}^{k}(-1)^{i+1} P w_{i}(x)}
$$


Then, using that if $x \in A_{j}$ then we can write

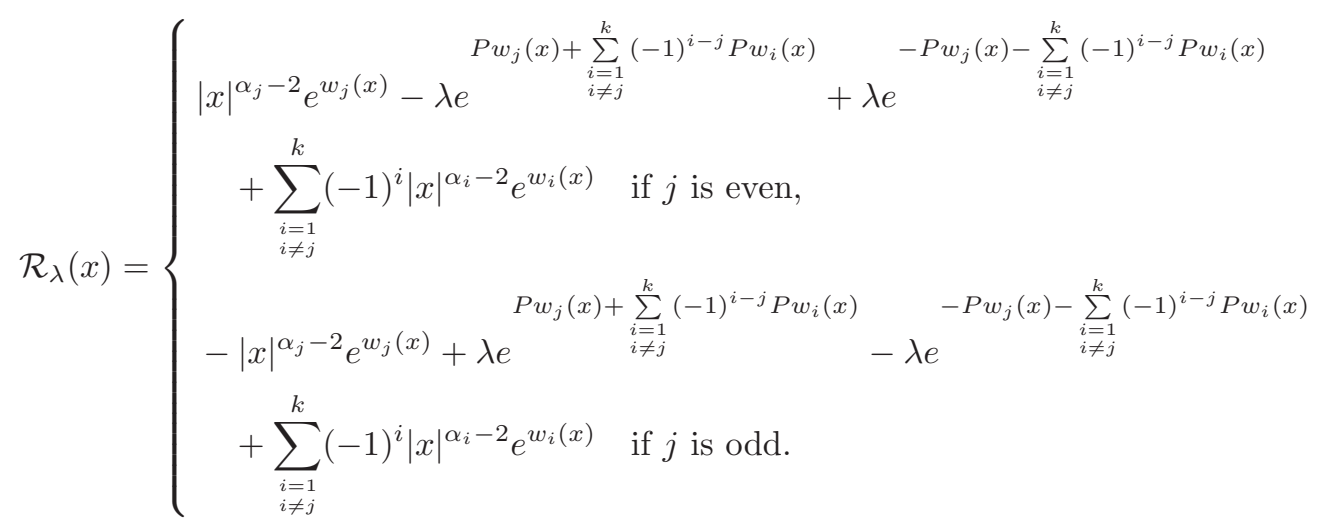

we have

$$
\begin{aligned}
& \int_{\Omega}\left|\mathcal{R}_{\lambda}(x)\right|^{p} d x=\sum_{j=1}^{k} \int_{A_{j}}\left|R_{\lambda}(x)\right|^{p} d x \\
& \leq\left. C \sum_{j=1}^{k} \int_{A_{j}}|| x\right|^{\alpha_{j}-2} e^{w_{j}(x)}-\left.\lambda e^{P w_{j}(x)+\sum_{\substack{i=1 \\
i \neq j}}^{k}(-1)^{i-j} P w_{i}(x)}\right|^{p} d x \\
& +C \sum_{j=1}^{k} \int_{A_{j}}\left|\lambda e^{-P w_{j}(x)+\sum_{\substack{i=1 \\
i \neq j}}^{k}(-1)^{i-j+1} P w_{i}(x)}\right|^{p} d x \\
& +\left.\left.C \sum_{\substack{i, j=1 \\
i \neq j}}^{k} \int_{A_{j}}|| x\right|^{\alpha_{i}-2} e^{w_{i}(x)}\right|^{p} d x=: I_{1}+I_{2}+I_{3} .
\end{aligned}
$$

Let us estimate $I_{1}$. For any $j=1, \ldots, k$ we have

$$
\begin{aligned}
& \left.\int_{A_{j}}|| x\right|^{\alpha_{j}-2} e^{w_{j}(x)}-\left.\lambda e^{P w_{j}(x)+\sum_{\substack{i=1 \\
i \neq j}}^{k}(-1)^{i-j} P w_{i}(x)}\right|^{p} d x \\
& =\int_{A_{j}}|x|^{\left(\alpha_{j}-2\right) p} e^{p w_{j}(x)}\left|1-e^{P w_{j}(x)-w_{j}(x)-\left(\alpha_{j}-2\right) \ln |x|+\sum_{i \neq j}(-1)^{i-j} P w_{i}(x)+\ln \lambda}\right|^{p} d x \\
& =C \delta_{j}^{2-2 p} \int_{\frac{A_{j}}{\delta_{j}}} \frac{|y|^{\left(\alpha_{j}-2\right) p}}{\left(1+|y|^{\alpha_{j}}\right)^{2 p}}\left|1-e^{P w_{j}\left(\delta_{j} y\right)-w_{j}\left(\delta_{j} y\right)-\left(\alpha_{j}-2\right) \ln \left|\delta_{j} y\right|+\sum_{i \neq j}(-1)^{i-j} P w_{i}\left(\delta_{j} y\right)+\ln \lambda}\right|^{p} d y
\end{aligned}
$$

(we use that $e^{t}-1=e^{\theta t} t$ for some $\theta \in(0,1)$ and we use Lemma 2.2.)

$$
\begin{aligned}
& =O\left(\delta_{j}^{2-2 p} \int_{\frac{A_{j}}{\delta_{j}}} \frac{|y|^{\left(\alpha_{j}-2\right) p}}{\left(1+|y|^{\left.\alpha_{j}\right)^{2 p}}\right.}\left|\Theta_{j}(y)\right|^{p} d y\right)= \\
& =O\left(\delta_{j}^{2-2 p} \int_{\frac{A_{j}}{\delta_{j}}} \frac{|y|^{\left(\alpha_{j}-2\right) p}}{\left(1+|y|^{\left.\alpha_{j}\right)^{2 p}}\right.}\left|\delta_{j}\right| y|+\lambda|^{p} d y\right)=O\left(\delta_{j}^{2-2 p} \lambda^{p}\right)+O\left(\delta_{j}^{2-p}\right) \\
& =O\left(\delta_{1}^{2-2 p} \lambda^{p}\right)+O\left(\delta_{k}^{2-p}\right)=O\left(\lambda^{p+(1-p)(2 k-1)}\right)+O\left(\lambda^{\frac{2-p}{2(2 k-1)}}\right)=
\end{aligned}
$$


$=O\left(\lambda^{\frac{2-p}{2(2 k-1)}}\right)$

provided $p$ is close enough to 1 . Therefore, we get

$$
I_{1}=O\left(\lambda^{\frac{2-p}{2(2 k-1)}}\right) \text {. }
$$

Let us estimate $I_{2}$. For any $j=1, \ldots, k$,

$$
\begin{aligned}
& \int_{A_{j}}\left|\lambda e^{-P w_{j}(x)+\sum_{\substack{i=1 \\
i \neq j}}^{k}(-1)^{i-j+1} P w_{i}(x)}\right|^{p} d x
\end{aligned}
$$

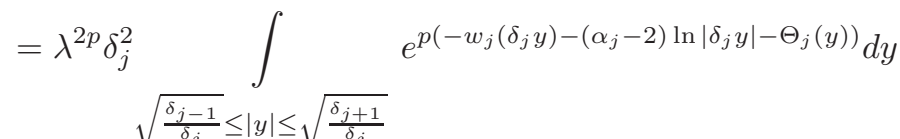

$$
\begin{aligned}
& =C \lambda^{2 p} \delta_{j}^{2+2 p} \int_{\sqrt{\frac{\delta_{j-1}}{\delta_{j}}} \leq|y| \leq \sqrt{\frac{\delta_{j+1}}{\delta_{j}}}} \frac{\left(1+|y|^{\alpha_{j}}\right)^{2 p}}{|y|^{\left(\alpha_{j}-2\right) p}} e^{-p \Theta_{j}(y)} d y \\
& =O\left(\lambda^{2 p} \delta_{j}^{2+2 p} \int_{\sqrt{\frac{\delta_{j-1}}{\delta_{j}}} \leq|y| \leq \sqrt{\frac{\delta_{j+1}}{\delta_{j}}}} \frac{\left(1+|y|^{\alpha_{j}}\right)^{2 p}}{|y|^{\left(\alpha_{j}-2\right) p}} d y\right)
\end{aligned}
$$

(we agree that $\delta_{0}=0$ and $\delta_{k+1}=+\infty$ )

$$
=O\left(\lambda^{2 p} \delta_{j}^{2+2 p}\left[\left(\frac{\delta_{j+1}}{\delta_{j}}\right)^{p \frac{\alpha_{j}+2}{2}+1}+\left(\frac{\delta_{j}}{\delta_{j-1}}\right)^{p \frac{\alpha_{j}-2}{2}-1}\right]\right)
$$

(if $j=1$ only the first term appears, while if $j=k$ only the second term appears)

$$
=O\left(\lambda^{2 p} \delta_{j}^{2+2 p}\left[\left(\frac{\delta_{j+1}}{\delta_{j}}\right)^{2 j p-1}+\left(\frac{\delta_{j}}{\delta_{j-1}}\right)^{(2 j-2) p-1}\right]\right)
$$

(since the best rate is obtained as $j=2$ )

$$
\begin{aligned}
& =O\left(\lambda^{2 p} \delta_{2}^{2+2 p}\left[\left(\frac{\delta_{3}}{\delta_{2}}\right)^{4 p+1}+\left(\frac{\delta_{2}}{\delta_{1}}\right)^{2 p-1}\right]\right) \\
& \left(\text { since } \frac{2 k}{15}(4 p+1)<\frac{2 k}{3}(2 p-1)\right) \\
& =O\left(\lambda^{2 p+\frac{2 k-3}{3}(1+p)-\frac{2 k}{3}(2 p-1)}\right)=O\left(\lambda^{\frac{3 p-3+2 k}{3}}\right) .
\end{aligned}
$$

Therefore, we get

$$
I_{2}=O\left(\lambda^{\frac{2 k}{3}}\right)
$$

Let us estimate $I_{3}$. For any $i, j=1, \ldots, k$ with $i \neq j$ we have

$$
\begin{aligned}
& \int_{A_{j}}\left|\frac{|x|^{\alpha_{i}-2}}{\left(\delta_{i}^{\alpha_{i}}+|x|^{\alpha_{i}}\right)^{2}}\right|^{p} d x \\
& \left(\text { we scale } x=\delta_{i} y\right) \\
& =C \delta_{i}^{2-2 p} \int_{\frac{\sqrt{\delta_{j-1} \delta_{j}}}{\delta_{i}} \leq|y| \leq \frac{\sqrt{\delta_{j} \delta_{j+1}}}{\delta_{i}}} \frac{|y|^{\left(\alpha_{i}-2\right) p}}{\left(1+|y|^{\alpha_{i}}\right)^{2 p}} d y
\end{aligned}
$$




$$
\begin{aligned}
& =\left\{\begin{array}{c}
O\left(\delta_{i}^{2-2 p}\left(\frac{\sqrt{\delta_{j} \delta_{j+1}}}{\delta_{i}^{2}}\right)^{\left(\alpha_{i}-2\right) p+2}\right)=O\left(\delta_{i}^{2-2 p}\left(\frac{\delta_{j}}{\delta_{j+1}}\right)^{(2 i-2) p+1}\right) \\
\text { if } j=1, \ldots, k-1 \text { and } i>j, \\
O\left(\delta_{i}^{2-2 p}\left(\frac{\delta_{i}}{\sqrt{\delta_{j-1} \delta_{j}}}\right)^{-\left(\alpha_{i}+2\right) p+2}\right)=O\left(\delta_{i}^{2-2 p}\left(\frac{\delta_{j-1}}{\delta_{j}}\right)^{2 i p-1}\right) \\
\text { if } j=2, \ldots, k \text { and } i<j . \\
O\left(\delta_{2}^{2-2 p}\left(\frac{\delta_{1}}{\delta_{2}}\right)^{2 p+1}\right)=O\left(\lambda^{\frac{2 k p+4 k+3(p-1)}{3}}\right)=O\left(\lambda^{2 k}\right), \\
O\left(\delta_{1}^{2-2 p}\left(\frac{\delta_{1}}{\delta_{2}}\right)^{2 p-1}\right)=O\left(\lambda^{\frac{-2 k p+4 k+3(p-1)}{3}}\right)=O\left(\lambda^{\frac{2}{3} k+(1-p)\left(\frac{2}{3} k-1\right)}\right)
\end{array}\right. \\
& =
\end{aligned}
$$

Therefore, if $p$ is close enough to 1 we get

$$
I_{3}=O\left(\lambda^{\frac{2}{3} k+(1-p)\left(\frac{2}{3} k-1\right)}\right) .
$$

Lemma 3.2. Let $\mathcal{S}_{\lambda}$ as in (3.2). There exists $\epsilon>0$ such that for any $p \in[1,1+\epsilon)$ we have

$$
\left\|\mathcal{S}_{\lambda}\right\|_{p}=O\left(\lambda^{\frac{2-p}{2 p(2 k-1)}}\right) .
$$

Proof. By 2.15) we get

$$
\begin{aligned}
& \int_{\Omega}\left|\mathcal{S}_{\lambda}(x)\right|^{p} d x=\sum_{j=1}^{k} \int_{A_{j}}\left|\mathcal{S}_{\lambda}(x)\right|^{p} d x \\
& =O\left(\sum_{j=1}^{k} \int_{A_{j}}\left|\lambda f^{\prime}\left(W_{\lambda}\right)(x)-2 \alpha_{j}^{2} \frac{|x|^{\alpha_{j}-2}}{\left(\delta_{j}^{\alpha_{j}}+|x|^{\alpha_{j}}\right)^{2}}\right|^{p} d x\right) \\
& +O\left(\sum_{\substack{i, j=1 \\
i \neq j}}^{k} \int_{A_{j}}\left|2 \alpha_{i}^{2} \frac{|x|^{\alpha_{i}-2}}{\left(\delta_{i}^{\alpha_{i}}+|x|^{\alpha_{i}}\right)^{2}}\right|^{p} d x\right):=J_{1}+J_{2}
\end{aligned}
$$

The integral $J_{2}$ was estimated in (3.9):

$$
J_{2}=O\left(\lambda^{\frac{2}{3} k+(1-p)\left(\frac{2}{3} k-1\right)}\right) .
$$

Let us estimated $J_{1}$. For any $j=1, \ldots, k$, we will scale $x=\delta_{j} y$. We observe that by (2.7)

$$
W_{\lambda}\left(\delta_{j} y\right)=(-1)^{j}\left[\Theta_{j}(y)+w_{j}\left(\delta_{j} y\right)+\left(\alpha_{j}-2\right) \ln \left|\delta_{j} y\right|-\ln \lambda\right]
$$

and so

$$
\begin{aligned}
& \lambda f^{\prime}\left(W_{\lambda}\left(\delta_{j} y\right)\right) \\
& =\lambda e^{(-1)^{j}\left[\Theta_{j}(y)+w_{j}\left(\delta_{j} y\right)+\left(\alpha_{j}-2\right) \ln \left|\delta_{j} y\right|-\ln \lambda\right]}+\lambda e^{(-1)^{j+1}\left[\Theta_{j}(y)+w_{j}\left(\delta_{j} y\right)+\left(\alpha_{j}-2\right) \ln \left|\delta_{j} y\right|-\ln \lambda\right]} \\
& =e^{\left[\Theta_{j}(y)+w_{j}\left(\delta_{j} y\right)+\left(\alpha_{j}-2\right) \ln \left|\delta_{j} y\right|\right]}+\lambda^{2} e^{-\left[\Theta_{j}(y)+w_{j}\left(\delta_{j} y\right)+\left(\alpha_{j}-2\right) \ln \left|\delta_{j} y\right|\right]} \\
& =\frac{2 \alpha_{j}^{2}}{\delta_{j}^{2}} \frac{|y|^{\alpha_{j}-2}}{\left(1+|y|^{\alpha_{j}}\right)^{2}} e^{\Theta_{j}(y)}+\lambda^{2} \delta_{j}^{2} \frac{\left(1+|y|^{\alpha_{j}}\right)^{2}}{2 \alpha_{j}^{2}|y|^{\alpha_{j}-2}} e^{-\Theta_{j}(y)},
\end{aligned}
$$


from which we deduce taking also into account Lemma 2.2

$$
\begin{aligned}
& \int_{A_{j}}\left|\lambda f^{\prime}\left(W_{\lambda}\right)(x)-2 \alpha_{j}^{2} \frac{|x|^{\alpha_{j}-2}}{\left(\delta_{j}^{\alpha_{j}}+|x|^{\alpha_{j}}\right)^{2}}\right|^{p} d x \\
& =\delta_{j}^{2} \int_{\frac{A_{j}}{\delta_{j}}}\left|\lambda f^{\prime}\left(W_{\lambda}\left(\delta_{j} y\right)\right)-\frac{2 \alpha_{j}^{2}}{\delta_{j}^{2}} \frac{|y|^{\alpha_{j}-2}}{\left(1+|y|^{\alpha_{j}}\right)^{2}}\right|^{p} d y \\
& =O\left(\delta_{j}^{2-2 p} \int_{\frac{A_{j}}{\delta_{j}}}\left|2 \alpha_{j}^{2} \frac{|y|^{\alpha_{j}-2}}{\left(1+|y|^{\alpha_{j}}\right)^{2}}\left(e^{\Theta_{j}(y)}-1\right)\right|^{p} d y\right) \\
& +O\left(\lambda^{2 p} \delta_{j}^{2+2 p} \int_{\frac{A_{j}}{\delta_{j}}}\left|\frac{\left(1+|y|^{\alpha_{j}}\right)^{2}}{2 \alpha_{j}^{2}|y|^{\alpha_{j}-2}} e^{-\Theta_{j}(y)}\right|^{p} d y\right) \\
& =O\left(\delta_{j}^{2-2 p} \int_{\frac{A_{j}}{\delta_{j}}}\left|\frac{|y|^{\alpha_{j}-2}}{\left(1+|y|^{\alpha_{j}}\right)^{2}} \Theta_{j}(y)\right|^{p} d y\right) \\
& +O\left(\lambda^{2 p} \delta_{j}^{2+2 p} \int_{\frac{A_{j}}{\delta_{j}}}\left|\frac{\left(1+|y|^{\alpha_{j}}\right)^{2}}{|y|^{\alpha_{j}-2}}\right|^{p} d y\right)
\end{aligned}
$$

(the first term is estimated in (3.5) and the second term is estimated in (3.7))

$$
=O\left(\lambda^{\frac{2-p}{2(2 k-1)}}\right)+O\left(\lambda^{\frac{2}{3} k}\right) .
$$

Therefore, we get

$$
J_{1}=O\left(\lambda^{\frac{2-p}{2(2 k-1)}}\right)+O\left(\lambda^{\frac{2}{3} k}\right) .
$$

Finally, the claim follows collecting all the previous estimates.

\section{THE LINEAR THEORY}

Let us consider the linear operator

$$
\mathcal{L}_{\lambda}(\phi):=-\Delta \phi-\left(\sum_{i=1}^{k} 2 \alpha_{i}^{2} \frac{|x|^{\alpha_{i}-2}}{\left(\delta_{i}^{\alpha_{i}}+|x|^{\alpha_{i}}\right)^{2}}\right) \phi .
$$

Let us study the invertibility of the linearized operator $\mathcal{L}_{\lambda}$.

Proposition 4.1. For any $p>1$ there exists $\lambda_{0}>0$ and $c>0$ such that for any $\lambda \in\left(0, \lambda_{0}\right)$ and for any $h \in \mathrm{L}^{p}(\Omega)$ there exists a unique $\phi \in \mathrm{W}^{2,2}(\Omega)$ solution of

$$
\mathcal{L}_{\lambda}(\phi)=\psi \text { in } \Omega, \phi=0 \text { on } \partial \Omega,
$$

which satisfies

$$
\|\phi\| \leq c|\ln \lambda|\|h\|_{p} .
$$

Proof. We argue by contradiction. Assume there exist $p>1$, sequences $\lambda_{n} \rightarrow 0, \psi_{n} \in \mathrm{L}^{\infty}(\Omega)$ and $\phi_{n} \in \mathrm{W}^{2,2}(\Omega)$ such that

$$
-\Delta \phi_{n}-\sum_{i=1}^{k} 2 \alpha_{i}^{2} \frac{\delta_{i_{n}}^{\alpha_{i}}|x|^{\alpha_{i}-2}}{\left(\delta_{i n}^{\alpha_{i}}+|x|^{\alpha_{i}}\right)^{2}} \phi_{n}=\psi_{n}, \text { in } \Omega, \phi_{n}=0 \text { on } \partial \Omega,
$$


with $\delta_{1 n}, \ldots, \delta_{k n}$ defined as in (2.5) and

$$
\left\|\phi_{n}\right\|=1 \quad \text { and } \quad\left|\ln \lambda_{n}\right|\left\|\psi_{n}\right\|_{p} \rightarrow 0 .
$$

For any $j=1, \ldots, k$ we define $\phi_{n}^{j}(y):=\phi_{n}\left(\delta_{i n} y\right)$ with $y \in \Omega_{n}^{j}:=\frac{\Omega}{\delta_{n}^{j}}$.

For sake of simplicity, in the following we will omit the index $n$ in all the sequences.

Step 1: we will show that

$$
\phi^{j}(y) \rightarrow \gamma_{j} \frac{1-|y|^{\alpha_{j}}}{1+|y|^{\alpha_{j}}} \text { for some } \gamma_{j} \in \mathbb{R} .
$$

weakly in $\mathrm{H}_{\alpha_{j}}\left(\mathbb{R}^{2}\right)$ and strongly in $\mathrm{L}_{\alpha_{j}}\left(\mathbb{R}^{2}\right.$ ) (see (6.4) and (6.4)).

First of all we claim that each $\phi^{j}$ is bounded in the space $\mathrm{H}_{\alpha_{j}}\left(\mathbb{R}^{2}\right)$ defined in (6.4).

Indeed, if we multiply (4.2) by $\phi$ we deduce that for any $j$

$$
\begin{aligned}
\int_{\Omega} 2 \alpha_{j}^{2} \frac{\delta_{j}^{\alpha_{j}}|x|^{\alpha_{j}-2}}{\left(\delta_{j}^{\alpha_{j}}+|x|^{\alpha_{j}}\right)^{2}} \phi^{2}(x) d x & \leq \sum_{i=1}^{k} \int_{\Omega} 2 \alpha_{i}^{2} \frac{\delta_{i}^{\alpha_{i}}|x|^{\alpha_{i}-2}}{\left(\delta_{i}^{\alpha_{i}}+|x|^{\alpha_{i}}\right)^{2}} \phi^{2}(x) d x \\
& =\int_{\Omega}|\nabla \phi(x)|^{2} d x-\int_{\Omega} \psi(x) \phi(x) d x \\
& =1+O\left(\|\psi\|_{p}\|\phi\|\right)=O(1)
\end{aligned}
$$

Our claim follows since by scaling

$$
\int_{\Omega^{j}}\left|\nabla \phi^{j}(y)\right|^{2} d y=\delta_{j}^{2} \int_{\Omega^{j}}\left|\nabla \phi\left(\delta_{j} y\right)\right|^{2} d y=\int_{\Omega}|\nabla \phi(x)|^{2} d x=1 .
$$

and

$$
\int_{\Omega^{j}} 2 \alpha_{j}^{2} \frac{|y|^{\alpha_{j}-2}}{\left(1+|y|^{\alpha_{j}}\right)^{2}}\left(\phi^{j}(y)\right)^{2} d y=\int_{\Omega} 2 \alpha_{j}^{2} \frac{\delta_{j}^{\alpha_{j}}|x|^{\alpha_{j}-2}}{\left(\delta_{j}^{\alpha_{j}}+|x|^{\alpha_{j}}\right)^{2}} \phi^{2}(x) d x .
$$

Therefore, by Proposition (6.1) we can assume that (up to a subsequence) $\phi^{j} \rightarrow \phi_{0}^{j}$ weakly in $\mathrm{H}_{\alpha_{j}}\left(\mathbb{R}^{2}\right)$ and strongly in $\mathrm{L}_{\alpha_{j}}\left(\mathbb{R}^{2}\right)$.

Now, we point out that each function $\phi^{j}$ solves the problem

$$
-\Delta \phi^{j}=2 \alpha_{j}^{2} \frac{|y|^{\alpha_{j}-2}}{\left(1+|y|^{\alpha_{j}}\right)^{2}} \phi^{j}+\rho_{j}(y) \phi^{j}+\delta_{j}^{2} \psi\left(\delta_{j} y\right) \text { in } \Omega^{j}, \phi^{j}=0 \text { on } \partial \Omega^{j},
$$

where

$$
\rho^{j}(y):=\sum_{\substack{i=1 \\ i \neq j}}^{k} 2 \alpha_{i}^{2} \frac{\delta_{i}^{\alpha_{i}} \delta_{j}^{\alpha_{j}}|y|^{\alpha_{i}-2}}{\left(\delta_{i}^{\alpha_{i}}+\delta_{j}^{\alpha_{i}}|y|^{\alpha_{i}}\right)^{2}} .
$$

Now, let $\varphi \in C_{0}^{\infty}\left(\mathbb{R}^{2}\right)$ be a given function and let $\mathcal{K}$ its support. It is clear that if $n$ is large enough

$$
\mathcal{K} \subset \frac{A_{j}}{\delta_{j}}=\left\{y \in \Omega^{j}: \sqrt{\frac{\delta_{j-1}}{\delta_{j}}} \leq|y| \leq \sqrt{\frac{\delta_{j+1}}{\delta_{j}}}\right\},
$$

where $A_{j}$ is the annulus defined in (2.15). We multiply equation (4.5) by $\varphi$ and we get

$$
\begin{aligned}
& \int_{\mathcal{K}} \nabla \phi^{j}(y) \nabla \varphi(y) d y-\int_{\mathcal{K}} 2 \alpha_{j}^{2} \frac{|y|^{\alpha_{j}-2}}{\left(1+|y|^{\alpha_{j}}\right)^{2}} \phi^{j}(y) \varphi(y) d y \\
& =\sum_{\substack{i=1 \\
i \neq j}}^{k} \int_{\mathcal{K}} 2 \alpha_{i}^{2} \frac{\delta_{i}^{\alpha_{i}} \delta_{j}^{\alpha_{j}}|y|^{\alpha_{i}-2}}{\left(\delta_{i}^{\alpha_{i}}+\delta_{j}^{\alpha_{j}}|y|^{\alpha_{i}}\right)^{2}} \phi^{j}(y) \varphi(y) d y+\int_{\mathcal{K}} \delta_{j}^{2} \psi\left(\delta_{j} y\right) \varphi(y) d y .
\end{aligned}
$$

Therefore, passing to the limit we get

$$
\int_{\mathcal{K}} \nabla \phi_{0}^{j}(y) \nabla \varphi(y) d y-\int_{\mathcal{K}} 2 \alpha_{j}^{2} \frac{|y|^{\alpha_{j}-2}}{\left(1+|y|^{\alpha_{j}}\right)^{2}} \phi_{0}^{j}(y) \varphi(y) d y=0 \forall \varphi \in C_{0}^{\infty}\left(\mathbb{R}^{2}\right),
$$


because

$$
\begin{aligned}
& \sum_{\substack{i=1 \\
i \neq j}}^{k} \int_{\mathcal{K}} 2 \alpha_{i}^{2} \frac{\delta_{i}^{\alpha_{i}} \delta_{j}^{\alpha_{j}}|y|^{\alpha_{i}-2}}{\left(\delta_{i}^{\alpha_{i}}+\delta_{j}{ }^{\alpha_{j}}|y|^{\alpha_{i}}\right)^{2}} \phi^{j}(y) \varphi(y) d y \\
& =O\left(\sum_{\substack{i=1 \\
i \neq j}}^{k} \int_{\frac{A}{j}_{j}} 2 \alpha_{i}^{2} \frac{\delta_{i}^{\alpha_{i}} \delta_{j}{ }^{\alpha_{j}}|y|^{\alpha_{i}-2}}{\left(\delta_{i}^{\alpha_{i}}+\delta_{j}{ }^{\alpha_{j}}|y|^{\left.\alpha_{i}\right)^{2}}\right.}\left|\phi^{j}(y)\right| d y\right) \quad\left(\text { because } \mathcal{K} \subset \frac{A_{j}}{\delta_{j}}\right) \\
& =O\left(\sum_{\substack{i=1 \\
i \neq j}}^{k} \int_{A_{j}} 2 \alpha_{i}^{2} \frac{\delta_{i}^{\alpha_{i}}|x|^{\alpha_{i}-2}}{\left(\delta_{i}^{\alpha_{i}}+|x|^{\alpha_{i}}\right)^{2}}|\phi(x)| d x\right) \quad\left(\text { we scale } x=\delta_{j} y\right) \\
& =O\left(\sum_{\substack{i=1 \\
i \neq j}}^{k}\left(\int_{A_{j}}\left|2 \alpha_{i}^{2} \frac{\delta_{i}^{\alpha_{i}}|x|^{\alpha_{i}-2}}{\left(\delta_{i}{ }^{\alpha_{i}}+|x|^{\alpha_{i}}\right)^{2}} d x\right|^{p}\right)^{1 / p}\|\phi\|_{q}\right) \text { (we use Hölder's estimate) } \\
& =o(1) \text { (we use estimate (3.9) and the fact that }|\phi|_{q} \leq 1 \text { ) }
\end{aligned}
$$

and

$$
\int_{\mathcal{K}} \delta_{j}^{2} \psi\left(\delta_{j} y\right) \varphi(y) d y=O\left(\int_{\Omega^{j}} \delta_{j}^{2}\left|\psi\left(\delta_{j} y\right)\right| d y\right)=O\left(\int_{\Omega}|\psi(x)| d x\right)=O\left(\|\psi\|_{p}\right)=o(1) .
$$

By (4.7) we deduce that $\phi_{0}^{j}$ is a solution to the equation

$$
-\Delta \phi_{0}^{j}=2 \alpha_{j}^{2} \frac{|y|^{\alpha_{j}-2}}{\left(1+|y|^{\alpha_{j}}\right)^{2}} \phi_{0}^{j} \text { in } \mathbb{R}^{2} \backslash\{0\} .
$$

Finally, since $\int_{\mathbb{R}^{2}}\left|\nabla \phi_{0}^{j}(y)\right|^{2} d y \leq 1$ it is standard to see that $\phi_{0}^{j}$ is a solution in the whole space $\mathbb{R}^{2}$. By Theorem 6.1 we get the claim.

Step 2: we will show that $\gamma_{j}=0$ for any $j=1, \ldots, k$.

Here we are inspired by some ideas used by Gladiali-Grossi [17.

We set

$$
\sigma_{i}(\lambda):=\ln \lambda \int_{\Omega^{i}} 2 \alpha_{i}^{2} \frac{|y|^{\alpha_{i}-2}}{\left(1+|y|^{\alpha_{i}}\right)^{2}} \phi^{i}(y) d y .
$$

We will show that

$$
\sigma_{i}:=\lim _{\lambda \rightarrow 0} \sigma_{i}(\lambda)=0 \text { for any } i=1, \ldots, k .
$$

We know that $\phi$ solves the problem (see [4.5)

$$
-\Delta \phi=\sum_{j=1}^{k} 2 \alpha_{j}^{2} \frac{\delta_{j}^{\alpha_{j}}|x|^{\alpha_{j}-2}}{\left(\delta_{j}^{\alpha_{j}}+|x|^{\alpha_{j}}\right)^{2}} \phi+\psi \text { in } \Omega, \phi=0 \text { on } \partial \Omega .
$$

Set $Z_{i}(x):=\frac{\delta_{i}^{\alpha_{i}}-|x|^{\alpha_{i}}}{\delta_{i}^{\alpha_{i}}+|x|^{\alpha_{i}}}$. We know that $Z_{i}$ solves (see Theorem 6.1)

$$
-\Delta Z_{i}=2 \alpha_{i}^{2} \frac{\delta_{i}^{\alpha_{i}}|x|^{\alpha_{i}-2}}{\left(\delta_{i}^{\alpha_{i}}+|x|^{\alpha_{i}}\right)^{2}} Z_{i} \quad \text { in } \mathbb{R}^{2}
$$

Let $P Z_{i}$ be its projection onto $\mathrm{H}_{0}^{1}(\Omega)$ (see (2.2) ), i.e.

$$
-\Delta P Z_{i}=2 \alpha_{i}^{2} \frac{\delta_{i}^{\alpha_{i}}|x|^{\alpha_{i}-2}}{\left(\delta_{i}^{\alpha_{i}}+|x|^{\alpha_{i}}\right)^{2}} Z_{i} \text { in } \Omega, P Z_{i}=0 \text { on } \partial \Omega
$$

By maximum principle (see also Lemma 2.1) we deduce that

$$
P Z_{i}(x)=Z_{i}(x)+1+O\left(\delta_{i}^{\alpha_{i}}\right)=\frac{2 \delta_{i}^{\alpha_{i}}}{\delta_{i}^{\alpha_{i}}+|x|^{\alpha_{i}}}+O\left(\delta_{i}^{\alpha_{i}}\right)
$$


frow which we get

$$
P Z_{i}\left(\delta_{j} y\right)=\left\{\begin{array}{l}
O\left(\frac{1}{|y|^{\alpha_{i}}}\left(\frac{\delta_{i}}{\delta_{j}}\right)^{\alpha_{i}}\right)+O\left(\delta_{i}^{\alpha_{i}}\right) \text { if } i<j, \\
\frac{2}{1+|y|^{\alpha_{i}}}+O\left(\delta_{i}^{\alpha_{i}}\right) \text { if } i=j, \\
2+O\left(|y|^{\alpha_{i}}\left(\frac{\delta_{j}}{\delta_{i}}\right)^{\alpha_{i}}\right)+O\left(\delta_{i}^{\alpha_{i}}\right) \text { if } i>j .
\end{array}\right.
$$

Now, we multiply (4.10) by $(\ln \lambda) P Z_{i}$ and (4.11) by $(\ln \lambda) \phi$. If we subtract the two equations obtained, we get

$$
\begin{aligned}
\ln \lambda \int_{\Omega} 2 \alpha_{i}^{2} \frac{\delta_{i}^{\alpha_{i}}|x|^{\alpha_{i}-2}}{\left(\delta_{i}^{\alpha_{i}}+|x|^{\alpha_{i}}\right)^{2}} \phi(x) Z_{i}(x) d x= & \ln \lambda \sum_{j=1}^{k} \int_{\Omega} 2 \alpha_{j}^{2} \frac{\delta_{j}^{\alpha_{j}}|x|^{\alpha_{j}-2}}{\left(\delta_{j}^{\alpha_{j}}+|x|^{\alpha_{j}}\right)^{2}} \phi(x) P Z_{i}(x) d x \\
& +\ln \lambda \int_{\Omega} \psi(x) P Z_{i}(x) d x
\end{aligned}
$$

and so

$$
\begin{aligned}
& \ln \lambda \int_{\Omega} 2 \alpha_{i}^{2} \frac{\delta_{j}^{\alpha_{i}}|x|^{\alpha_{i}-2}}{\left(\delta_{i}^{\alpha_{i}}+|x|^{\alpha_{i}}\right)^{2}} \phi(x)\left(P Z_{i}(x)-Z_{i}(x)\right) d x \\
& +\ln \lambda \sum_{\substack{j=1 \\
j \neq i}}^{k} 2 \alpha_{\Omega}^{2} \frac{\delta_{j}^{\alpha_{j}}|x|^{\alpha_{j}-2}}{\left(\delta_{j}^{\alpha_{j}}+|x|^{\alpha_{j}}\right)^{2}} \phi(x) P Z_{i}(x) d x \\
& +\ln \lambda \int_{\Omega} \psi(x) P Z_{i}(x) d x=0 .
\end{aligned}
$$

We are going to pass to the limit in 4.14).

The last term is

$$
\ln \lambda \int_{\Omega} \psi(x) P Z_{i}(x) d x=O\left(|\ln \lambda|\|\psi\|_{p}\right)=o(1)
$$

because of (4.3) and since by (4.12) we get $\left\|P Z_{i}\right\|_{\infty}=O(1)$.

The first term is

$$
\ln \lambda \int_{\Omega} 2 \alpha_{i}^{2} \frac{\delta_{i}^{\alpha_{i}}|x|^{\alpha_{i}-2}}{\left(\delta_{i}^{\alpha_{i}}+|x|^{\alpha_{i}}\right)^{2}} \phi(x)\left(P Z_{i}(x)-Z_{i}(x)\right) d x
$$

(we scale $x=\delta_{i} y$ and we apply (4.12))

$$
=\ln \lambda \int_{\Omega^{i}} 2 \alpha_{i}^{2} \frac{|y|^{\alpha_{i}-2}}{\left(1+|y|^{\alpha_{i}}\right)^{2}} \phi^{i}(y) d y+O\left(\delta_{i}^{\alpha_{i}}|\ln \lambda| \int_{\Omega^{i}} 2 \alpha_{i}^{2} \frac{|y|^{\alpha_{i}-2}}{\left(1+|y|^{\alpha_{i}}\right)^{2}}\left|\phi^{i}(y)\right| d y\right)
$$

(we use (4.8) and (4.4))

$$
=\sigma_{i}(\lambda)+o(1) \text {. }
$$


We estimate the second term. If $j \neq i$ we get

$$
\begin{aligned}
& \left.\ln \lambda \int_{\Omega} 2 \alpha_{j}^{2} \frac{\delta_{j}^{\alpha_{j}}|x|^{\alpha_{j}-2}}{\left(\delta_{j}^{\alpha_{j}}+|x|^{\alpha_{j}}\right)^{2}} \phi(x) P Z_{i}(x) d x \text { (we scale } x=\delta_{j} y\right) \\
& =\ln \lambda \int_{\Omega^{j}} 2 \alpha_{j}^{2} \frac{|y|^{\alpha_{j}-2}}{\left(1+|y|^{\alpha_{j}}\right)^{2}} \phi^{j}(y) P Z_{i}\left(\delta_{j} y\right) d y \text { (we use (4.13)) } \\
& \int 2 \ln \lambda \int_{\Omega^{j}} 2 \alpha_{j}^{2} \frac{|y|^{\alpha_{j}-2}}{\left(1+|y|^{\alpha_{j}}\right)^{2}} \phi^{j}(y) d y+ \\
& = \begin{cases}+O\left(|\ln \lambda| \int_{\Omega^{j}} 2 \alpha_{j}^{2} \frac{|y|^{\alpha_{j}-2}}{\left(1+|y|^{\alpha_{j}}\right)^{2}}\left|\phi^{j}(y)\right|\left(|y|^{\alpha_{i}}\left(\frac{\delta_{j}}{\delta_{i}}\right)^{\alpha_{i}}+\delta_{i}^{\alpha_{i}}\right) d y\right) & \text { if } j<i \\
O\left(|\ln \lambda| \int_{\Omega^{j}} 2 \alpha_{j}^{2} \frac{|y|^{\alpha_{j}-2}}{\left(1+|y|^{\alpha_{j}}\right)^{2}}\left|\phi^{j}(y)\right|\left(\frac{1}{|y|^{\alpha_{i}}}\left(\frac{\delta_{i}}{\delta_{j}}\right)^{\alpha_{i}}+\delta_{i}^{\alpha_{i}}\right) d y\right) & \text { if } j>i .\end{cases}
\end{aligned}
$$

(we use (4.8), (4.18), (4.19) and (4.20) )

$$
= \begin{cases}2 \sigma_{j}(\lambda)+o(1) & \text { if } j<i \\ o(1) & \text { if } j>i .\end{cases}
$$

By (4.14), (4.15), (4.16) and (4.17) we get

$$
\sigma_{1}(\lambda)=o(1) \text { and } \sigma_{i}(\lambda)+2 \sum_{j=1}^{i-1} \sigma_{j}(\lambda)=o(1) \text { for any } i=2, \ldots, k,
$$

which implies passing to the limit and using the definition of $\sigma_{i}$ given in (4.9),

$$
\sigma_{1}=0 \text { and } \sigma_{i}+2 \sum_{j=1}^{i-1} \sigma_{j}=0 \text { for any } i=2, \ldots, k \text {. }
$$

Therefore, (4.9) immediately follows.

We used the following three estimates. If $j<i$ we have

$$
\begin{aligned}
& \left(|\ln \lambda| \frac{\delta_{j}}{\delta_{i}}\right)^{\alpha_{i}} \int_{\Omega^{j}} \frac{|y|^{\alpha_{j}+\alpha_{i}-2}}{\left(1+|y|^{\alpha_{j}}\right)^{2}}\left|\phi^{j}(y)\right| d y \text { (by Hölder's inequality) } \\
& =O\left(|\ln \lambda|\left(\frac{\delta_{j}}{\delta_{i}}\right)^{\alpha_{i}} \delta_{j}^{\frac{2(1-p)}{p}}\|\phi\|\left(\int_{\mathbb{R}^{2}}\left(\frac{|y|^{\alpha_{j}+\alpha_{i}-2}}{\left(1+|y|^{\alpha_{j}}\right)^{2}}\right)^{p} d y\right)^{1 / p}\right) \\
& =O\left(|\ln \lambda|\left(\frac{\delta_{j}}{\delta_{i}}\right)^{\alpha_{i}} \delta_{j}^{\frac{2(1-p)}{p}}\right)=o(1)
\end{aligned}
$$

and if $j>i$ we have

$$
\begin{aligned}
& \left(|\ln \lambda| \frac{\delta_{i}}{\delta_{j}}\right)^{\alpha_{i}} \int_{\Omega^{j}} \frac{1}{|y|^{\alpha_{i}-\alpha_{j}+2}\left(1+|y|^{\left.\alpha_{j}\right)^{2}}\right.}\left|\phi^{j}(y)\right| d y \text { (by Hölder's inequality) } \\
& =O\left(|\ln \lambda|\left(\frac{\delta_{j}}{\delta_{i}}\right)^{\alpha_{i}} \delta_{j}^{\frac{2(1-p)}{p}}\|\phi\|\left(\int_{\mathbb{R}^{2}}\left(\frac{1}{|y|^{\alpha_{i}-\alpha_{j}+2}\left(1+|y|^{\left.\alpha_{j}\right)^{2}}\right.}\right)^{1 / p} d y\right)^{1 / p}\right) \\
& \quad\left(\text { we use } \alpha_{i}>\alpha_{j} \text { and we choose } p \text { close to } 1\right) \\
& =O\left(|\ln \lambda|\left(\frac{\delta_{j}}{\delta_{i}}\right)^{\alpha_{i}} \delta_{j}^{\frac{2(1-p)}{p}}\right)=o(1) ;
\end{aligned}
$$


moreover for any $i$ and $j$ we have

$$
\begin{aligned}
& |\ln \lambda| \delta_{i}^{\alpha_{i}} \int_{\Omega^{j}} \frac{|y|^{\alpha_{j}-2}}{\left(1+|y|^{\alpha_{j}}\right)^{2}}\left|\phi^{j}(y)\right| d y \text { (by Hölder's inequality) } \\
& =O\left(|\ln \lambda| \delta_{i}^{\alpha_{i}} \delta_{j}^{\frac{2(1-p)}{p}}\|\phi\|\left(\int_{\mathbb{R}^{2}}\left(\frac{|y|^{\alpha_{j}-2}}{\left(1+|y|^{\left.\alpha_{j}\right)^{2}}\right.}\right)^{p} d y\right)^{1 / p}\right) \\
& \quad(\text { we choose } p \text { close to } 1) \\
& =O\left(|\ln \lambda| \delta_{i}^{\alpha_{i}} \delta_{j}^{\frac{2(1-p)}{p}}\right)=o(1) .
\end{aligned}
$$

Finally, we have all the ingredients to show that

$$
\gamma_{i}=0 \text { for any } i=1, \ldots, k \text {. }
$$

We know that $P w_{i}$ solves the problem

$$
-\Delta P w_{i}=2 \alpha_{i}^{2} \frac{\delta_{i}^{\alpha_{i}}|x|^{\alpha_{i}-2}}{\left(\delta_{i}^{\alpha_{i}}+|x|^{\alpha_{i}}\right)^{2}} \text { in } \Omega, P w_{i}=0 \text { on } \partial \Omega .
$$

Now, we multiply (4.10) by $P w_{i}$ and (4.22) by $\phi$. If we subtract the two equations obtained, we get

$$
\begin{aligned}
\int_{\Omega} 2 \alpha_{i}^{2} \frac{\delta_{i}^{\alpha_{i}}|x|^{\alpha_{i}-2}}{\left(\delta_{i}^{\alpha_{i}}+|x|^{\alpha_{i}}\right)^{2}} \phi(x) d x= & \sum_{j=1}^{k} \int_{\Omega} 2 \alpha_{j}^{2} \frac{\delta_{j}^{\alpha_{j}}|x|^{\alpha_{j}-2}}{\left(\delta_{j}^{\alpha_{j}}+|x|^{\alpha_{j}}\right)^{2}} \phi(x) P w_{i}(x) d x \\
& +\int_{\Omega} \psi(x) P w_{i}(x) d x .
\end{aligned}
$$

We want to pass to the limit in 4.23).

The L.H.S. of 4.23) reduces to

$$
\begin{aligned}
& \int_{\Omega} 2 \alpha_{i}^{2} \frac{\delta_{i}^{\alpha_{i}}|x|^{\alpha_{i}-2}}{\left(\delta_{i}^{\alpha_{i}}+|x|^{\alpha_{i}}\right)^{2}} \phi(x) d x\left(\text { we scale } x=\delta_{i} y\right) \\
& =\int_{\Omega^{i}} 2 \alpha_{i}^{2} \frac{|y|^{\alpha_{i}-2}}{\left(1+|y|^{\alpha_{i}}\right)^{2}} \phi^{i}(y) d y=o(1) \text { (because of (4.29) and (4.4)). }
\end{aligned}
$$

The last term of the R.H.S. of 4.23) gives

$$
\int_{\Omega} \psi(x) P w_{i}(x) d x=O\left(|\ln \lambda|\|\psi\|_{p}\right) o(1),
$$

because of (4.3) and since by (2.16) we get $\left\|P w_{i}\right\|_{\infty}=O(|\ln \lambda|)$.

Finally, we claim that the first term of the R.H.S. of (4.23) is

$$
\begin{aligned}
& \sum_{j=1}^{k} \int_{\Omega} 2 \alpha_{j}^{2} \frac{\delta_{j}^{\alpha_{j}}|x|^{\alpha_{j}-2}}{\left(\delta_{j}^{\alpha_{j}}+|x|^{\alpha_{j}}\right)^{2}} \phi(x) P w_{i}(x) d x \\
& =\left\{\begin{array}{lr}
4 \pi \alpha_{i}\left(\begin{array}{lr}
\gamma_{i}+2 \sum_{j=i+1}^{k} \gamma_{j} \\
4 \pi \alpha_{k} \gamma_{k}+o(1) & \text { if } i=1, \ldots, k-1,
\end{array}\right.
\end{array}\right.
\end{aligned}
$$

Therefore, passing to the limit, by (4.23), (4.24), 4.25) and (4.26) we immediately get

$$
\gamma_{k}=0 \text { and } \gamma_{i}+2 \sum_{j=i+1}^{k} \gamma_{j}=0 \text { for any } i=1, \ldots, k-1
$$

which implies (4.21). 
It only remains to prove (4.26). We have

$$
\begin{aligned}
& \int_{\Omega} 2 \alpha_{j}^{2} \frac{\delta_{j}^{\alpha_{j}}|x|^{\alpha_{j}-2}}{\left(\delta_{j}^{\alpha_{j}}+|x|^{\alpha_{j}}\right)^{2}} \phi(x) P w_{i}(x) d x\left(\text { we scale } x=\delta_{j} y\right) \\
& \left.=\int_{\Omega^{j}} 2 \alpha_{j}^{2} \frac{|y|^{\alpha_{j}-2}}{\left(1+|y|^{\alpha_{j}}\right)^{2}} \phi^{j}(y) P w_{i}\left(\delta_{j} y\right) d y \text { (we use (2.17) }\right)
\end{aligned}
$$

$$
\left\{\begin{array}{c}
\int_{\Omega^{j}} 2 \alpha_{j}^{2} \frac{|y|^{\alpha_{j}-2}}{\left(1+|y|^{\alpha_{j}}\right)^{2}} \phi^{j}(y)\left(-2 \alpha_{i} \ln \delta_{i}+h_{i}(0)\right) d y+ \\
+O\left(\int_{\Omega^{j}} 2 \alpha_{j}^{2} \frac{|y|^{\alpha_{j}-2}}{\left(1+|y|^{\alpha_{j}}\right)^{2}}\left|\phi^{j}(y)\right|\left(|y|^{\alpha_{i}}\left(\frac{\delta_{j}}{\delta_{i}}\right)^{\alpha_{i}}+\delta_{j}|y|+\delta_{i}^{\alpha_{i}}\right) d y\right) \quad \text { if } j<i \\
\int_{\Omega^{i}} 2 \alpha_{i}^{2} \frac{|y|^{\alpha_{i}-2}}{\left(1+|y|^{\alpha_{i}}\right)^{2}} \phi^{i}(y)\left(-2 \alpha_{i} \ln \delta_{i}-2 \ln \left(1+|y|^{\alpha_{i}}\right)+h_{i}(0)\right) d y+ \\
+O\left(\int_{\Omega^{i}} 2 \alpha_{i}^{2} \frac{|y|^{\alpha_{i}-2}}{\left(1+|y|^{\alpha_{i}}\right)^{2}}\left|\phi^{i}(y)\right|\left(\delta_{i}|y|+\delta_{i}^{\alpha_{i}}\right) d y\right) \\
\int_{\Omega^{j}} 2 \alpha_{j}^{2} \frac{|y|^{\alpha_{j}-2}}{\left(1+|y|^{\alpha_{j}}\right)^{2}} \phi^{j}(y)\left(-2 \alpha_{i} \ln \left(\delta_{j}|y|\right)+h_{i}(0)\right) d y+ \\
+O\left(\int_{\Omega^{j}} 2 \alpha_{j}^{2} \frac{|y|^{\alpha_{j}-2}}{\left(1+|y|^{\alpha_{j}}\right)}\left|\phi^{j}(y)\right|\left(\frac{1}{|y|^{\alpha_{i}}}\left(\frac{\delta_{i}}{\delta_{j}}\right)^{\alpha_{i}}+\delta_{j}|y|+\delta_{i}^{\alpha_{i}}\right) d y\right)
\end{array}\right.
$$

(we use the relation between $\delta_{i}$ and $\lambda$ in (2.5) and we use (4.18), (4.19), (4.20) and (4.28)

$$
\left\{\begin{array}{l}
\int_{\Omega^{j}} 2 \alpha_{j}^{2} \frac{|y|^{\alpha_{j}-2}}{\left(1+|y|^{\alpha_{j}}\right)^{2}} \phi^{j}(y)\left[-2 \alpha_{i} \ln d_{i}-2(2(k-i)+1) \ln \lambda+h_{i}(0)\right] d y \\
\quad+o(1) \text { if } j<i \\
\int_{\Omega^{i}} 2 \alpha_{i}^{2} \frac{|y|^{\alpha_{i}-2}}{\left(1+|y|^{\alpha_{i}}\right)^{2}} \phi^{j}(y)\left[-2 \alpha_{i} \ln d_{i}-2(2(k-i)+1) \ln \lambda-2 \ln \left(1+|y|^{\alpha_{i}}\right)+h_{i}(0)\right] d y \\
\quad+o(1) \text { if } j=i \\
\int_{\Omega^{j}} 2 \alpha_{j}^{2} \frac{|y|^{\alpha_{j}-2}}{\left(1+|y|^{\alpha_{j}}\right)^{2}} \phi^{j}(y)\left[-2 \alpha_{i} \ln d_{j}-2(2(k-j)+1) \ln \lambda-2 \alpha_{i} \ln |y|+h_{i}(0)\right] d y \\
\quad+o(1) \text { if } j>i
\end{array}\right.
$$

(we use the definition of $\sigma_{i}$ in (4.8) and we use (4.4) and (4.29) 


$$
=\left\{\begin{array}{c}
-2(2(k-i)+1) \sigma_{j}(\lambda)+o(1) \\
\text { if } j<i \\
-2(2(k-i)+1) \sigma_{i}(\lambda)+\int_{\Omega^{i}} 2 \alpha_{i}^{2} \frac{|y|^{\alpha_{i}-2}}{\left(1+|y|^{\alpha_{i}}\right)^{2}} \phi^{i}(y)\left[-2 \ln \left(1+|y|^{\alpha_{i}}\right)\right] d y+o(1) \\
\text { if } j=i \\
-2(2(k-j)+1) \sigma_{j}(\lambda)+\int_{\Omega^{j}} 2 \alpha_{j}^{2} \frac{|y|^{\alpha_{j}-2}}{\left(1+|y|^{\alpha_{j}}\right)^{2}} \phi^{j}(y)\left[-2 \alpha_{i} \ln |y|\right] d y+o(1) \\
\text { if } j>i
\end{array}\right.
$$

(we use (4.9) and (4.4) because $\ln \left(1+|y|^{\alpha_{j}}\right), \ln |y| \in \mathrm{L}_{\alpha_{j}}\left(\mathbb{R}^{2}\right)$ )

$$
= \begin{cases}o(1) & \text { if } j<i \\ \gamma_{i} \int_{\mathbb{R}^{2}} 2 \alpha_{i}^{2} \frac{|y|^{\alpha_{i}-2}}{\left(1+|y|^{\alpha_{i}}\right)^{2}} \frac{1-|y|^{\alpha_{i}}}{1+|y|^{\alpha_{i}}}\left[-2 \ln \left(1+|y|^{\alpha_{i}}\right)\right] d y+o(1) & \text { if } j=i \\ \gamma_{j} \int_{\mathbb{R}^{2}} 2 \alpha_{j}^{2} \frac{|y|^{\alpha_{j}-2}}{\left(1+|y|^{\alpha_{j}}\right)^{2}} \frac{1-|y|^{\alpha_{j}}}{1+|y|^{\alpha_{j}}}\left[-2 \alpha_{i} \ln |y|\right] d y+o(1) & \text { if } j>i\end{cases}
$$

(we use (4.30) and 4.31)

$$
= \begin{cases}o(1) & \text { if } j<i \\ 4 \pi \alpha_{i} \gamma_{i}+o(1) & \text { if } j=i \\ 8 \pi \alpha_{i} \gamma_{j}+o(1) & \text { if } j>i\end{cases}
$$

If we sum (4.27) over the index $j$ we get (4.26).

We used the following estimate. For any $j$ we have

$$
\begin{aligned}
& \delta_{j} \int_{\Omega^{j}} \frac{|y|^{\alpha_{j}-1}}{\left(1+|y|^{\left.\alpha_{j}\right)^{2}}\right.}\left|\phi^{j}(y)\right| d y \text { (by Hölder's inequality) } \\
& =O\left(\delta_{j} \delta_{j}^{\frac{2(1-p)}{p}}\|\phi\|\left(\int_{\mathbb{R}^{2}}\left(\frac{|y|^{\alpha_{j}-1}}{\left(1+|y|^{\left.\alpha_{j}\right)^{2}}\right.}\right)^{p} d y\right)^{1 / p}\right) \\
& \quad(\text { we choose } p \text { close to } 1) \\
& =O\left(\delta_{j}^{\frac{2-p}{p}}\right)=o(1) .
\end{aligned}
$$


A straightforward computation leads to

$$
\begin{aligned}
& \int_{\Omega} 2 \alpha_{i}^{2} \frac{|y|^{\alpha_{i}-2}}{\left(1+|y|^{\alpha_{i}}\right)^{2}} \frac{1-|y|^{\alpha_{i}}}{1+|y|^{\alpha_{i}}} d y=0, \\
& \int_{\Omega} 2 \alpha_{i}^{2} \frac{|y|^{\alpha_{i}-2}}{\left(1+|y|^{\alpha_{i}}\right)^{2}} \frac{1-|y|^{\alpha_{i}}}{1+|y|^{\alpha_{i}}} \ln \left(1+|y|^{\alpha_{i}}\right)^{2} d y=-4 \pi \alpha_{i}, \\
& \int_{\Omega} 2 \alpha_{i}^{2} \frac{|y|^{\alpha_{i}-2}}{\left(1+|y|^{\alpha_{i}}\right)^{2}} \frac{1-|y|^{\alpha_{i}}}{1+|y|^{\alpha_{i}}} \ln |y| d y=-4 \pi .
\end{aligned}
$$

Step 3: we will show that a contradiction arises! We multiply equation (4.2) by $\phi$ and we get

$$
\begin{aligned}
1 & =\sum_{i=1}^{k} \int_{\Omega} 2 \alpha_{i}^{2} \frac{\delta_{i}^{\alpha_{i}}|x|^{\alpha_{i}-2}}{\left(\delta_{i}{ }^{\alpha_{i}}+|x|^{\alpha_{i}}\right)^{2}} \phi^{2}(x) d x+\int_{\Omega} \psi(x) \phi(x) d x \\
& =\sum_{i=1}^{k} \int_{\Omega^{i}} 2 \alpha_{i}^{2} \frac{|y|^{\alpha_{i}-2}}{\left(1+|y|^{\alpha_{i}}\right)^{2}}\left(\phi^{i}(y)\right)^{2} d y+O\left(\|\psi\|_{p}\|\phi\|\right) \quad \text { (we use (4.3)) } \\
& =\sum_{i=1}^{k} \int_{\Omega^{i}} 2 \alpha_{i}^{2} \frac{|y|^{\alpha_{i}-2}}{\left(1+|y|^{\alpha_{i}}\right)^{2}}\left(\phi^{i}(y)\right)^{2} d y+o(1) \\
& \left.=o(1) \text { (because } \phi^{i} \rightarrow 0 \text { strongly in } \mathrm{L}_{\alpha_{i}}\left(\mathbb{R}^{2}\right)\right)
\end{aligned}
$$

and a contradiction arises!

\section{A contraction mapping argument and the proof of the main theorem}

First of all we point out that $W_{\lambda}+\phi_{\lambda}$ is a solution to (1.1) if and only if $\phi_{\lambda}$ is a solution of the problem

$$
\mathcal{L}_{\lambda}(\phi)=\mathcal{N}_{\lambda}(\phi)+\mathcal{S}_{\lambda} \phi+\mathcal{R}_{\lambda} \text { in } \Omega
$$

where the error term $\mathcal{R}_{\lambda}$ is defined in (3.1), the linear error term $\mathcal{S}_{\lambda}$ is defined in (3.2) the linear operator $\mathcal{L}_{\lambda}$ is defined in (4.1) and and the higher order term $\mathcal{N}_{\lambda}$ is defined as

$$
\mathcal{N}_{\lambda}(\phi):=\lambda\left[f\left(W_{\lambda}+\phi\right)-f\left(W_{\lambda}\right)-f^{\prime}\left(W_{\lambda}\right) \phi\right] .
$$

Proposition 5.1. If $p$ is close enough to 1 there exist $\lambda_{0}>0$ and $R>0$ such that for any $\lambda \in\left(0, \lambda_{0}\right)$ there exists a unique solution $\phi_{\lambda} \in \mathrm{H}_{0}^{1}(\Omega)$ to

$$
-\Delta\left(W_{\lambda}+\phi_{\lambda}\right)=\lambda f\left(W_{\lambda}+\phi_{\lambda}\right) \text { in } \Omega, \quad \phi=0 \text { on } \partial \Omega,
$$

such that $\phi(x)=\phi(-x)$ for any $x \in \Omega$ and

$$
\left\|\phi_{\lambda}\right\| \leq R \lambda^{\frac{2-p}{2 p(2 k-1)}}|\ln \lambda| .
$$

Proof. Let $\mathcal{H}:=\left\{\phi \in \mathrm{H}_{0}^{1}(\Omega): \phi(x)=\phi(-x) \forall x \in \Omega\right\}$. As a consequence of Proposition 4.1. we conclude that $\phi$ is a solution to (5.3) if and only if it is a fixed point for the operator $\mathcal{T}_{\lambda}: \mathcal{H} \rightarrow \mathcal{H}$, defined by

$$
T_{\lambda}(\phi)=\left(\mathcal{L}_{\lambda}\right)^{-1}\left(\mathcal{N}_{\lambda}(\phi)+\mathcal{S}_{\lambda} \phi+\mathcal{R}_{\lambda}\right),
$$

where $\mathcal{L}_{\lambda}, \mathcal{N}_{\lambda}, \mathcal{S}_{\lambda}$ and $\mathcal{R}_{\lambda}$ are defined in (3.1), (5.2), (3.2) and (3.1), respectively.

Let us introduce the ball $B_{\lambda, R}:=\left\{\phi \in \mathcal{H}:\|\phi\| \leq R \lambda^{\frac{2-p}{2 p(2 k-1)}}\right\}$. We will show that $T_{\lambda}: B_{\lambda, R} \rightarrow B_{\lambda, R}$ is a contraction mapping provided $\lambda$ is small enough and $r$ is large enough.

Let us prove that $T_{\lambda}$ maps the ball $B_{\lambda, r}$ into itself, i.e.

$$
\|\phi\| \leq R \lambda^{\frac{2-p}{2 p(2 k-1)}}|\ln \lambda| \Longrightarrow\left\|\mathcal{T}_{\lambda}(\phi)\right\| \leq R \lambda^{\frac{2-p}{2 p(2 k-1)}}|\ln \lambda| .
$$


By Lemma 5.1 (where we take $h=\mathcal{N}_{\lambda}(\phi)+\mathcal{S}_{\lambda} \phi+\mathcal{R}_{\lambda}$ ), we deduce that:

$$
\left\|\mathcal{T}_{\lambda}(\phi)\right\| \leq c|\ln \lambda|\left(\left\|\mathcal{N}_{\lambda}(\phi)\right\|_{p}+\left\|\mathcal{S}_{\lambda} \phi\right\|_{p}+\left\|\mathcal{R}_{\lambda}\right\|_{p}\right)
$$

(we use (5.6) with $p$ and $r$ close enough to 1 for the first term, we use Hölder's inequality for the second term and we use Lemma 3.1 for the third term)

$$
\begin{aligned}
& \leq c|\ln \lambda|\left(\|\phi\|^{2} e^{c_{2}\|\phi\|^{2}} \lambda^{(2 k-1) \frac{1-p r}{p r}}+\left\|\mathcal{S}_{\lambda}\right\|_{p q}\|\phi\|_{p s}+\lambda^{\frac{2-p}{2 p(2 k-1)}}\right) \\
& \leq c|\ln \lambda|\left(\|\phi\|^{2} e^{c_{2}\|\phi\|^{2}} \lambda^{(2 k-1) \frac{1-p r}{p r}}+\lambda^{\frac{2-p q}{2 p q(2 k-1)}}\|\phi\|+\lambda^{\frac{2-p}{2 p(2 k-1)}}\right)
\end{aligned}
$$

(we use Lemma 3.2)

and if we choose $q$ close enough to $1, R$ suitable large and $\lambda$ small enough we get (5.4).

Let us prove that $T_{\lambda}$ is a contraction mapping, i.e. there exists $L>1$ such that

$$
\|\phi\| \leq R \lambda^{\frac{2-p}{2 p(2 k-1)}}|\log \rho| \Longrightarrow\left\|\mathcal{T}_{\lambda}\left(\phi_{1}\right)-\mathcal{T}_{\lambda}\left(\phi_{2}\right)\right\| \leq L\left\|\phi_{1}-\phi_{2}\right\| .
$$

By Lemma 5.1 (where we take $h=\mathcal{N}_{\lambda}\left(\phi_{1}\right)-\mathcal{N}_{\lambda}\left(\phi_{2}\right)+\mathcal{S}_{\lambda}\left(\phi_{1}-\phi_{2}\right)$ ), we deduce that:

$$
\left\|\mathcal{T}_{\lambda}(\phi)\right\| \leq c|\ln \lambda|\left(\left\|\mathcal{N}_{\lambda}\left(\phi_{1}\right)-\mathcal{N}_{\lambda}\left(\phi_{2}\right)\right\|_{p}+\left\|\mathcal{S}_{\lambda}\left(\phi_{1}-\phi_{2}\right)\right\|_{p}\right)
$$

(we use (5.7) with $p$ and $r$ close enough to 1 for the first term and we use Hölder's inequality for the second term)

$$
\begin{aligned}
& \leq c|\ln \lambda|\left[c_{1} e^{c_{2}\left(\left\|\phi_{1}\right\|^{2}+\left\|\phi_{2}\right\|^{2}\right)} \lambda^{(2 k-1) \frac{1-p r}{p r}}\left\|\phi_{1}-\phi_{2}\right\|\left(\left\|\phi_{1}\right\|+\left\|\phi_{2}\right\|\right)\right. \\
& \left.+\left\|\mathcal{S}_{\lambda}\right\|_{p q}\left\|\phi_{1}-\phi_{2}\right\|_{p s}\right] \\
& \leq c|\ln \lambda|\left[c_{1} e^{c_{2}\left(\left\|\phi_{1}\right\|^{2}+\left\|\phi_{2}\right\|^{2}\right)} \lambda^{(2 k-1) \frac{1-p r}{p r}}\left(\left\|\phi_{1}\right\|+\left\|\phi_{2}\right\|\right)+\lambda^{\frac{2-p q}{2 p q(2 k-1)}}\right]\left\|\phi_{1}-\phi_{2}\right\|
\end{aligned}
$$

and if we choose $q$ close enough to $1, R$ suitable large and $\lambda$ small enough we get (5.5).

Lemma 5.1. For any $p \geq 1$ and $r>1$ there exist $\lambda_{0}>0$ and $c_{1}, c_{2}>0$ such that for any $\lambda \in\left(0, \lambda_{0}\right)$ we have for any $\phi, \phi_{1}, \phi_{2} \in \mathrm{H}_{0}^{1}(\Omega)$ :

$$
\left\|\mathcal{N}_{\lambda}(\phi)\right\|_{p} \leq c_{1} e^{c_{2}\|\phi\|^{2}} \lambda^{(2 k-1) \frac{1-p r}{p r}}\|\phi\|^{2}
$$

and

$$
\left\|\mathcal{N}_{\lambda}\left(\phi_{1}\right)-\mathcal{N}_{\lambda}\left(\phi_{2}\right)\right\|_{p} \leq c_{1} e^{c_{2}\left(\left\|\phi_{1}\right\|^{2}+\left\|\phi_{2}\right\|^{2}\right)} \lambda^{(2 k-1) \frac{1-p r}{p r}}\left\|\phi_{1}-\phi_{2}\right\|\left(\left\|\phi_{1}\right\|+\left\|\phi_{2}\right\|\right) .
$$

Proof Let us remark that (5.6) follows by choosing $\phi_{2}=0$ in (5.7) . Let us prove (5.7). We point out that

$$
\mathcal{N}_{\lambda}(\phi)=\lambda e^{W_{\lambda}}\left(e^{\phi}-1-\phi\right)-\lambda e^{-W_{\lambda}}\left(e^{-\phi}-1+\phi\right)
$$

and so

$$
\mathcal{N}_{\lambda}\left(\phi_{1}\right)-\mathcal{N}_{\lambda}\left(\phi_{2}\right)=\underbrace{\lambda e^{W_{\lambda}}\left(e^{\phi_{1}}-e^{\phi_{2}}-\phi_{1}+\phi_{2}\right)}_{I_{1}}-\underbrace{\lambda e^{-W_{\lambda}}\left(e^{-\phi_{1}}-e^{-\phi_{2}}+\phi_{1}-\phi_{2}\right)}_{I_{2}} .
$$

We estimate $\left\|I_{1}\right\|_{p}$. The estimate of $\left\|I_{2}\right\|_{p}$ is similar.

By the mean value theorem, we easily deduce that

$$
\left|e^{a}-e^{b}-a+b\right| \leq e^{|a|+|b|}|a-b|(|a|+|b|) \text { for any } a, b \in \mathbb{R} .
$$


Therefore, we have

$$
\begin{aligned}
& \left\|I_{1}\right\|_{p}=\left(\int_{\Omega} \lambda^{p} e^{p W_{\lambda}}\left|e^{\phi_{1}}-e^{\phi_{2}}-\phi_{1}+\phi_{2}\right|^{p} d x\right)^{1 / p} \\
& \leq c \sum_{j=1}^{2}\left(\int_{\Omega} \lambda^{p} e^{p W_{\lambda}} e^{p\left|\phi_{1}\right|+p\left|\phi_{2}\right|}\left|\phi_{1}-\phi_{2}\right|^{p}\left|\phi_{j}\right|^{p} d x\right)^{1 / p}
\end{aligned}
$$

(we use Hölder's inequality with $\frac{1}{r}+\frac{1}{s}+\frac{1}{t}=1$ )

$$
\leq c \sum_{j=1}^{2}\left(\int_{\Omega} \lambda^{p r} e^{p r W_{\lambda}} d x\right)^{1 /(p r)}\left(\int_{\Omega} e^{p s\left|\phi_{1}\right|+p s\left|\phi_{2}\right|} d x\right)^{1 /(p s)}\left(\int_{\Omega}\left|\phi_{1}-\phi_{2}\right|{ }^{p t}\left|\phi_{j}\right|^{p t} d x\right)^{1 /(p t)}
$$

(we use Lemma 5.2)

$$
\leq c \sum_{j=1}^{2}\left(\int_{\Omega} \lambda^{p r} e^{p r W_{\lambda}} d x\right)^{1 /(p r)} e^{(p s) /(8 \pi)\left(\left|\phi_{1}\right|^{2}+\left|\phi_{2}\right|^{2}\right)}\left\|\phi_{1}-\phi_{2}\right\|\left\|\phi_{j}\right\| .
$$

We have to estimate

$$
\int_{\Omega} \lambda^{p r} e^{p r W_{\lambda}(x)} d x=\sum_{j} \int_{A_{j}} \lambda^{p r} e^{p r W_{\lambda}(x)} d x,
$$

where $A_{j}$ is the annulus defined in (2.15).

If $j$ is even we get

$$
\begin{aligned}
& \int_{A_{j}} \lambda^{p r} e^{p r W_{\lambda}(x)} d x \text { (we use (2.7)) } \\
& =\delta_{j}^{2} \lambda^{p r} \int_{\frac{A_{j}}{\delta_{j}}} e^{p r\left[w_{j}\left(\delta_{j} y\right)+\left(\alpha_{j}-2\right) \ln \left|\delta_{j} y\right|-\ln \lambda+\Theta_{j}(y)\right]} d y \\
& \left.=\delta_{j}^{2-2 p r} \int_{\frac{A_{j}}{\delta_{j}}}\left(2 \alpha_{j}^{2} \frac{|y|^{\alpha_{j}-2}}{\left(1+|y|^{\alpha_{j}}\right)^{2}}\right)^{p r} e^{p r \Theta_{j}(y)} d y \text { (we use Lemma (․2.) }\right) \\
& =O\left(\delta_{j}^{2-2 p r}\right)=O\left(\lambda^{(2 k-1)(1-p r)}\right) \quad\left(\text { because } \delta_{j} \geq \delta_{1}=O\left(\lambda^{\frac{2 k-1}{2}}\right) \text { and } p r>1\right),
\end{aligned}
$$

and $j$ is odd we get

$$
\begin{aligned}
& \int_{A_{j}} \lambda^{p r} e^{p r W_{\lambda}(x)} d x \text { (we use (2.7)) } \\
& =\delta_{j}^{2} \lambda^{p r} \int_{\frac{A_{j}}{\delta_{j}}} e^{-p r\left[w_{j}\left(\delta_{j} y\right)+\left(\alpha_{j}-2\right) \ln \left|\delta_{j} y\right|-\ln \lambda+\Theta_{j}(y)\right]} d y \\
& =\delta_{j}^{2+2 p r} \lambda^{2 p r} \int_{\frac{\delta_{j-1}}{\delta_{j}} \leq|y| \leq \sqrt{\frac{\delta_{j+1}}{\delta_{j}}}\left(\frac{\left(1+|y|^{\alpha_{j}}\right)^{2}}{2 \alpha_{j}^{2}|y|^{\alpha_{j}-2}}\right)^{p r} e^{-p r \Theta_{j}(y)} d y \text { (we use Lemma (2.2) ) }} \\
& =O\left(\delta_{j}^{2+2 p r} \lambda^{2 p r}\left[\left(\frac{\delta_{j+1}}{\delta_{j}}\right)^{p r \frac{\alpha_{j}+2}{2}+1}+\left(\frac{\delta_{j}}{\delta_{j-1}}\right)^{p r \frac{\alpha_{j}-2}{2}-1}\right]\right) \\
& =O\left(\lambda^{p r}\left[\frac{\delta_{j}^{p r+1}}{\delta_{j+1}^{p r-1}}+\frac{\delta_{j}^{p r+1}}{\delta_{j-1}^{p r-1}}\right]\right)=O\left(\lambda^{\left(\frac{2 k}{3}-1\right)(1-p r)}\right)=O\left(\lambda^{(2 k-1)(1-p r)}\right),
\end{aligned}
$$


because by (2.12) and (2.10) we get

$$
\begin{gathered}
\left(\frac{\delta_{j+1}}{\delta_{j}}\right)^{p r \frac{\alpha_{j}+2}{2}+1}=\frac{\delta_{j+1}^{p r \frac{\alpha_{j+1}}{2}}}{\delta_{j}^{p r \frac{\alpha_{j}}{2}}} \frac{\delta_{j+1}^{p r \frac{\alpha_{j}-\alpha_{j+1}}{2}+p r+1}}{\delta_{j}^{p r+1}}=O\left(\frac{1}{\lambda^{p r} \delta_{j}^{p r+1} \delta_{j+1}^{p r-1}}\right), \\
\left(\frac{\delta_{j}}{\delta_{j-1}}\right)^{p r \frac{\alpha_{j}-2}{2}-1}=\frac{\delta_{j}^{p r \frac{\alpha_{j}}{2}}}{\delta_{j-1}^{p r \frac{\alpha_{j-1}}{2}}} \frac{\delta_{j}^{-p r-1}}{\delta_{j-1}^{p r \frac{\alpha_{j}-\alpha_{j-1}}{2}-p r-1}}=O\left(\frac{1}{\lambda^{p r} \delta_{j}^{p r+1} \delta_{j-1}^{p r-1}}\right) . \\
\frac{\delta_{j}^{p r+1}}{\delta_{j+1}^{p r-1}}=\left(\frac{\delta_{j}}{\delta_{j+1}}\right)^{p r} \delta_{j} \delta_{j+1}=o(1)
\end{gathered}
$$

and

$$
\begin{aligned}
& \lambda^{p r} \frac{\delta_{j}^{p r+1}}{\delta_{j-1}^{p r-1}}=\lambda^{p r}\left(\frac{\delta_{j}}{\delta_{j-1}}\right)^{p r-1} \delta_{j}^{2}=O\left(\lambda^{p r}\left(\frac{\delta_{2}}{\delta_{1}}\right)^{p r-1} \delta_{k}^{2}\right) \\
& =O\left(\lambda^{p r+\frac{2 k}{3}(1-p r)+\frac{1}{2 k-1}}\right)=O\left(\lambda^{\left(\frac{2 k}{3}-1\right)(1-p r)+\frac{2 k}{2 k-1}}\right) .
\end{aligned}
$$

Therefore, by (5.8) we obtain that $\left\|I_{1}\right\|_{p}$ satisfies estimate (5.7).

We recall the following Moser-Trudinger inequality [22, 29,

Lemma 5.2. There exists $c>0$ such that for any bounded domain $\Omega$ in $\mathbb{R}^{2}$

$$
\int_{\Omega} e^{4 \pi u^{2} /\|u\|^{2}} d x \leq c|\Omega|, \text { for any } u \in \mathrm{H}_{0}^{1}(\Omega) .
$$

In particular, there exists $c>0$ such that for any $\eta \in \mathbb{R}$

$$
\int_{\Omega} e^{\eta u} \leq c|\Omega| e^{\frac{\eta^{2}}{16 \pi}\|u\|^{2}}, \text { for any } u \in \mathrm{H}_{0}^{1}(\Omega) .
$$

Proof of Theorem 1.1. By Proposition 5.1 we have that

$$
u_{\lambda}=W_{\lambda}+\phi_{\lambda}=\sum_{i=1}^{k}(-1)^{i} P w_{i}(x)+\phi_{\lambda}
$$

is a solution to (1.1).

Let us prove (1.4). By (2.16), we derive that

$$
P w_{i}(x)=4 \pi \alpha_{i} G(x, 0)+o(1) \text { pointwise in } \Omega \backslash\{0\},
$$

and so, by (5.9) and (2.14) we get,

$$
u_{\lambda}(x)=4 \pi \sum_{i=1}^{k}(-1)^{i} \alpha_{i} G(x, 0)+o(1)=(-1)^{k} 8 \pi k G(x, 0)+o(1) \text { pointwise in } \Omega \backslash\{0\}
$$

Moreover, for some $\theta \in(0,1)$ we have that

$$
\lambda e^{W_{\lambda}+\phi_{\lambda}}-\lambda e^{-W_{\lambda}-\phi_{\lambda}}=\lambda e^{W_{\lambda}}-\lambda e^{-W_{\lambda}}+\lambda e^{W_{\lambda}+\theta \phi_{\lambda}} \phi_{\lambda}+\lambda e^{-W_{\lambda}-\theta \phi_{\lambda}} \phi_{\lambda} .
$$

Let us fix a compact set $K \subset \Omega$ which does not contain the origin and let $q>1$. From (5.12) we deduce

$$
\begin{aligned}
& \left\|\lambda e^{W_{\lambda}+\phi_{\lambda}}-\lambda e^{-W_{\lambda}-\phi_{\lambda}}\right\|_{L^{q}(K)} \\
& =O\left(\left\|\lambda e^{W_{\lambda}}-\lambda e^{-W_{\lambda}}\right\|_{L^{q}(K)}\right)+O\left(\left\|\lambda e^{W_{\lambda}+\theta \phi_{\lambda}} \phi_{\lambda}+\lambda e^{-W_{\lambda}-\theta \phi_{\lambda}} \phi_{\lambda}\right\|_{L^{q}(K)}\right) \\
& \left.=O(1) \text { (because of the definition of } W_{\lambda} \text { and (15.10) }\right) \\
& \left.+O\left(\left\|\lambda e^{W_{\lambda}}\right\|_{L^{\infty}(K)}\right)\left\|\lambda e^{\theta \Phi_{\lambda}}\right\|_{L^{2 q}(K)}\|\phi\|_{L^{2 q}(K)}\right)+O\left(\left\|\lambda e^{-W_{\lambda}}\right\|_{L^{\infty}(K)}\left\|\lambda e^{-\theta \Phi_{\lambda}}\right\|_{L^{2 q}(K)}\|\phi\|_{L^{2 q}(K)}\right) \\
& =O(1) \text { (using (5.10), Lemma [5.2 and Proposition [5.1). }
\end{aligned}
$$

Hence, by (5.12) and (5.13), we derive that the R.H.S. of problem (1.1) is bounded in $L^{q}(K)$. Then standard results imply (1.4). 
Let us prove (1.5) and (1.6). We will only consider the case $m_{+}(0)$, since the other is similar. By the definition of $m_{+}(0)$ we get

$$
\begin{aligned}
m_{+}(0) & =\lim _{r \rightarrow 0} \lim _{\lambda \rightarrow 0} \int_{B(0, r)} \lambda e^{W_{\lambda}+\phi_{\lambda}}=\lim _{r \rightarrow 0} \lim _{\lambda \rightarrow 0} \int_{B(0, r)} \lambda e^{W_{\lambda}}\left(1+e^{\theta \phi_{\lambda}} \phi_{\lambda}\right) \\
& =\lim _{r \rightarrow 0} \lim _{\lambda \rightarrow 0}\left(J_{1, \lambda, r}+J_{2, \lambda, r}\right) .
\end{aligned}
$$

So we have that,

$$
\begin{aligned}
& J_{1, \lambda, r}=\int_{B(0, r)} \lambda e^{W_{\lambda}}=\int_{B(0, r)} \lambda e^{\sum_{i=1}^{k}(-1)^{i} P w_{i}(x)}=\sum_{j=1}^{k} \int_{A_{j}} \lambda e^{(-1)^{j} P w_{j}(x)+\sum_{\substack{i=1 \\
i \neq j}}^{k}(-1)^{i-j} P w_{i}(x)} d x \\
& \text { (using (2.15) } \\
& =\sum_{\substack{j=1 \\
j \text { even }}}^{k} \int_{A_{j}} \lambda e^{P w_{j}(x)+\sum_{\substack{i=1 \\
i \neq j}}^{k}(-1)^{i-j} P w_{i}(x)} d x+\sum_{\substack{j=1 \\
j \text { odd }}}^{k} \int_{A_{j}} \lambda e^{-P w_{j}(x)+\sum_{\substack{i=1 \\
i \neq j}}^{k}(-1)^{i-j} P w_{i}(x)} d x \\
& =\sum_{\substack{j=1 \\
j \text { even }}}^{k} \int_{\mathbb{R}^{2}}|x|^{\alpha_{j}-2} e^{w_{j}(x)} d x+o(1) \quad(\text { using } \text { (3.5) and } p=1 \text { ) } \\
& \left.=\sum_{\substack{j=1 \\
j \text { even }}}^{k} 4 \pi \alpha_{i}+o(1)(\text { we use } \underline{(5.14})\right) \\
& =\sum_{\substack{j=1 \\
j \text { even }}}^{k} 4 \pi(4 i-2)+o(1)(\text { we use (2.4) }) \\
& =\left\{\begin{array}{ll}
4 \pi k(k-1) & \text { if } k \text { is even } \\
4 \pi k(k+1) & \text { if } k \text { is odd }
\end{array}+o(1) .\right.
\end{aligned}
$$

Here we used a result of Chen-Li [4] which states the mass

$$
\int_{\mathbb{R}^{2}}|y|^{\alpha-2} e^{w^{\alpha}(x)} d x=4 \pi \alpha
$$

where

$$
w^{\alpha}(x):=\ln 2 \alpha^{2} \frac{1}{\left(1+|x|^{\alpha}\right)^{2}}, \quad x \in \mathbb{R}^{2}
$$

So

$$
\lim _{r \rightarrow 0} \lim _{\lambda \rightarrow 0} I_{1, \lambda, r}=\left\{\begin{array}{ll}
4 \pi k(k-1) & \text { if } k \text { is even } \\
4 \pi k(k+1) & \text { if } k \text { is odd }
\end{array} .\right.
$$

On the other hand, arguing exactly as in (5.8) we get (for some $\frac{1}{p}+\frac{1}{q}+\frac{1}{s}=1$ )

$$
J_{2, \lambda, r}=\int_{B(0, r)} \lambda e^{W_{\lambda}} e^{\theta \phi_{\lambda}} \phi_{\lambda}=O\left(\left\|\lambda e^{W_{\lambda}}\right\|_{p}\left\|\lambda e^{\theta \Phi_{\lambda}}\right\|_{q}\|\phi\|_{s}\right)=o(1) .
$$

So we have that

$$
\lim _{r \rightarrow 0} \lim _{\lambda \rightarrow 0} I_{2, \lambda, r}=0
$$

which ends the proof. 


\section{ApPEnDiX}

We have the following result.

Theorem 6.1. Assume $\frac{\alpha}{2}$ is odd. If $\phi$ satisfies

$$
\phi(y)=\phi(-y) \text { for any } y \in \mathbb{R}^{2}
$$

and solves the equation

$$
-\Delta \phi=2 \alpha^{2} \frac{|y|^{\alpha-2}}{\left(1+|y|^{\alpha}\right)^{2}} \phi \text { in } \mathbb{R}^{2}, \quad \int_{\mathbb{R}^{2}}|\nabla \phi(y)|^{2} d y<+\infty,
$$

then there exists $a \in \mathbb{R}$ such that

$$
\phi(y)=\gamma \frac{1-|y|^{\alpha}}{1+|y|^{\alpha}} \text { for some } \gamma \in \mathbb{R} .
$$

Proof. Del Pino-Esposito-Musso in 9 proved that all the bounded solutions to (6.2) are a linear combination of the following functions (which are written in polar coordinates)

$$
\phi_{0}(y):=\frac{1-|y|^{\alpha}}{1+|y|^{\alpha}}, \phi_{1}(y):=\frac{|y|^{\frac{\alpha}{2}}}{1+|y|^{\alpha}} \cos \frac{\alpha}{2} \theta, \phi_{2}(y):=\frac{|y|^{\frac{\alpha}{2}}}{1+|y|^{\alpha}} \sin \frac{\alpha}{2} \theta .
$$

We observe that $\phi_{0}$ always satisfies (6.1), while if $\frac{\alpha}{2}$ is odd the functions $\phi_{1}$ and $\phi_{2}$ do not satisfy (6.1). So, we just have to prove that any solution $\phi$ of (6.2) is actually a bounded solution, i.e. $\phi \in \mathrm{L}^{\infty}\left(\mathbb{R}^{2}\right)$. The claim will follow by $[9$.

Since $\phi$ is a solution in the sense of distribution to (6.2), from the boundedness of RHS in $L_{l o c}^{2}\left(\mathbb{R}^{2}\right)$ and by the regularity theory we get that $\phi \in L_{l o c}^{\infty}\left(\mathbb{R}^{2}\right)$.

In order to end the proof we have to show that $\phi$ is bounded near infinity. Let us consider the Kelvin transform of $\phi$, namely,

$$
z(x)=\phi\left(\frac{x}{|x|^{2}}\right)
$$

A straightforward computation gives

$$
\int_{\mathbb{R}^{2}}|\nabla z(y)|^{2} d y=\int_{\mathbb{R}^{2}}|\nabla \phi(y)|^{2} d y
$$

and

$$
-\Delta z=2 \alpha^{2} \frac{|y|^{\alpha-2}}{\left(1+|y|^{\alpha}\right)^{2}} z \text { in } \mathbb{R}^{2} .
$$

So we have that $z$ satisfies the same problem as $\phi$ and then $z \in L_{l o c}^{\infty}\left(\mathbb{R}^{2}\right)$. This implies that $\phi$ is bounded near infinity which ends the proof.

For any $\alpha \geq 2$ let us consider the Banach spaces

$$
\mathrm{L}_{\alpha}\left(\mathbb{R}^{2}\right):=\left\{u \in \mathrm{W}_{l o c}^{1,2}\left(\mathbb{R}^{2}\right):\left\|\frac{|y|^{\frac{\alpha-2}{2}}}{1+|y|^{\alpha}} u\right\|_{L^{2}\left(\mathbb{R}^{2}\right)}<+\infty\right\}
$$

and

$$
\mathrm{H}_{\alpha}\left(\mathbb{R}^{2}\right):=\left\{u \in \mathrm{W}_{l o c}^{1,2}\left(\mathbb{R}^{2}\right):\|\nabla u\|_{L^{2}\left(\mathbb{R}^{2}\right)}+\left\|\frac{|y|^{\frac{\alpha-2}{2}}}{1+|y|^{\alpha}} u\right\|_{\mathrm{L}^{2}\left(\mathbb{R}^{2}\right)}<+\infty\right\},
$$

endowed with the norms

$$
\|u\|_{\mathrm{L}_{\alpha}}:=\left\|\frac{|y|^{\frac{\alpha-2}{2}}}{1+|y|^{\alpha}} u\right\|_{\mathrm{L}^{2}\left(\mathbb{R}^{2}\right)} \text { and }\|u\|_{\mathrm{H}_{\alpha}}:=\left(\|\nabla u\|_{\mathrm{L}^{2}\left(\mathbb{R}^{2}\right)}^{2}+\left\|\frac{|y|^{\frac{\alpha-2}{2}}}{1+|y|^{\alpha}} u\right\|_{\mathrm{L}^{2}\left(\mathbb{R}^{2}\right)}^{2}\right)^{1 / 2} .
$$

Proposition 6.1. The embedding $i_{\alpha}: \mathrm{H}_{\alpha}\left(\mathbb{R}^{2}\right) \hookrightarrow \mathrm{L}_{\alpha}\left(\mathbb{R}^{2}\right)$ is compact. 
Proof. Firstly, let $\alpha=2$. If $\mathbb{S}^{2}$ denotes the unit sphere in $\mathbb{R}^{3}$ with the standard metric and $\pi: \mathbb{S}^{2} \rightarrow \mathbb{R}^{3}$ is the stereographic projection through the north pole, then the map $u \rightarrow u \circ \pi$ is an isometry from $\mathrm{L}_{2}$ into $\mathrm{L}^{2}\left(\mathbb{S}^{2}\right)$ and from $\mathrm{H}_{2}$ into $\mathrm{H}^{1}\left(\mathbb{S}^{2}\right)$ Hence, the claim follows directly from the compactness of the embedding $\mathrm{H}^{1}\left(\mathbb{S}^{2}\right) \hookrightarrow \mathrm{L}^{2}\left(\mathbb{S}^{2}\right)$. Now, let $\alpha \geq 2$. Let us define an operator $\mathfrak{T}_{\alpha}: \mathrm{L}_{\alpha}\left(\mathbb{R}^{2}\right) \rightarrow \mathrm{L}_{2}\left(\mathbb{R}^{2}\right)$ by $\mathfrak{T}_{\alpha}(u)=\bar{u}$ where the function $\bar{u}$ is defined in this way

$$
\bar{u}(z):=\hat{u}(|z|, \theta) \text {, where } \hat{u}(s, \theta):=\tilde{u}\left(s^{2 / \alpha}, \theta\right) \text { and } \tilde{u}(r, \theta):=u(r \cos \theta, r \sin \theta) .
$$

We will prove that

$$
\left\|\mathfrak{T}_{\alpha}\right\|_{\mathcal{L}\left(\mathrm{L}_{\alpha}\left(\mathbb{R}^{2}\right), \mathrm{L}_{2}\left(\mathbb{R}^{2}\right)\right)}=\frac{2}{\alpha} \quad \text { and } \quad \frac{2}{\alpha} \leq\left\|\mathfrak{T}_{\alpha}\right\|_{\mathcal{L}\left(\mathrm{H}_{\alpha}\left(\mathbb{R}^{2}\right), \mathrm{H}_{2}\left(\mathbb{R}^{2}\right)\right)} \leq \frac{\alpha}{2} .
$$

The compactness of the embedding for $\alpha \geq 2$ will follow immediately, because $i_{\alpha}=\mathfrak{T}_{\alpha}^{-1}$ 。 $i_{2} \circ \mathfrak{T}_{\alpha}$.

Let us prove (6.5) A direct computation shows that

$$
\begin{aligned}
\int_{\mathbb{R}^{2}} \frac{|y|^{\alpha-2}}{\left(1+|y|^{\alpha}\right)^{2}} u^{2}(y) d y & =\int_{0}^{2 \pi} \int_{0}^{+\infty} \frac{r^{\alpha-1}}{\left(1+r^{\alpha}\right)^{2}} \tilde{u}^{2}(r, \theta) d r d \theta=\frac{2}{\alpha} \int_{0}^{2 \pi} \int_{0}^{+\infty} \frac{s}{\left(1+s^{2}\right)^{2}} \hat{u}^{2}(s, \theta) d s d \theta \\
& =\frac{2}{\alpha} \int_{\mathbb{R}^{2}} \frac{1}{\left(1+|z|^{\alpha}\right)^{2}} \bar{u}^{2}(z) d z,
\end{aligned}
$$

which proves the first estimate in (6.5). Moreover, we also have

$$
\int_{\mathbb{R}^{2}}|\nabla u|^{2}(y) d y=\int_{0}^{2 \pi} \int_{0}^{+\infty} r\left[\left(\partial_{r} \tilde{u}\right)^{2}+\frac{\left(\partial_{\theta} \tilde{u}\right)^{2}}{r^{2}}\right] d r d \theta=\frac{2}{\alpha} \int_{0}^{2 \pi} \int_{0}^{+\infty} s\left\{\frac{\alpha^{2}}{4}\left(\partial_{s} \hat{u}\right)^{2}+\frac{\left(\partial_{\theta} \hat{u}\right)^{2}}{s^{2}}\right\} d s d \theta
$$

and so

$$
\frac{2}{\alpha} \int_{\mathbb{R}^{2}}|\nabla \bar{u}|^{2}(z) d z \leq \int_{\mathbb{R}^{2}}|\nabla u|^{2}(y) d y \leq \frac{\alpha}{2} \int_{\mathbb{R}^{2}}|\nabla \bar{u}|^{2}(z) d z
$$

which proves the second estimate in (6.5).

\section{REFERENCES}

[1] Baraket, S.; Pacard, F. Construction of singular limits for a semilinear elliptic equation in dimension 2. Calc. Var. Partial Diff. Equ. 6 (1998) no. 1, 1-38.

[2] Bartolucci, D.; Pistoia, A. Existence and qualitative properties of concentrating solutions for the sinhPoisson equation. IMA J. Appl. Math. 72 (2007) no. 6, 706-729.

[3] Bartsch, T.; Pistoia, A.; Weth, T. N-vortex equilibria for ideal fluids in bounded planar domains and new nodal solutions of the sinh-Poisson and the Lane-Emden-Fowler equations. Comm. Math. Phys. 297 (2010), no. 3, 653-686.

[4] Chen, W; Li, C. Qualitative properties of solutions to some nonlinear elliptic equations in $\mathbb{R}^{2}$. Duke Math. J. 71 (1993) 427-439.

[5] Chorin, A. J. Vorticity and turbulunce. Springer, New York (1994)

[6] Del Pino, M.; Dolbeault, J.; Musso, M. "Bubble-tower" radial solutions in the slightly supercritical Brezis-Nirenberg problem. J. Differential Equations193 (2003), no. 2, 280-306.

[7] Del Pino, M.; Dolbeault, J.; Musso, M. The Brezis-Nirenberg problem near criticality in dimension 3. J. Math. Pures Appl. (9) 83 (2004), no. 12, 1405-1456.

[8] Del Pino, M.; Dolbeault, J.; Musso, M. Multiple bubbling for the exponential nonlinearity in the slightly supercritical case. Comm. Pure Appl. Anal. 5 (2006), no. 3, 463-482.

[9] Del Pino, M.; Esposito, P.; Musso, M. Nondegeneracy of entire solutions of a singular Liouvillle equation. Proc. Amer. Math. Soc. 140 (2012), no. 2, 581-588.

[10] Del Pino, M.; Esposito, P.; Musso, M. Two-dimensional Euler flows with concentrated vorticities. Trans. Amer. Math. Soc. 362 (2010), no. 12, 6381-6395.

[11] Del Pino, M.; Kowalczyk, M.; Musso, M. Singular limits in Liouville-type equations. Calc. Var. Partial Diff. Equ. 24 (2005) no. 1, 47-81.

[12] Del Pino, M.; Musso, M.; Pistoia, A. Super-critical boundary bubbling in a semilinear Neumann problem. Ann. Inst. H. Poincaré Anal. Non Linéaire 22 (2005), no. 1, 45-82.

[13] Esposito, P.; Grossi, M.; Pistoia, A. On the existence of blowing-up solutions for a mean field equation. Ann. IHP Anal. Non Lineaire 22 (2005) no. 2, 127-157. 
[14] Esposito, P.; Wei, J. Non-simple blow-up solutions for the Neumann two-dimensional sinh-Gordon equation. Calc. Var. Partial Differential Equations 34 (2009), no. 3, 341-375.

[15] Ge, Y.; Jing, R.; Pacard, F. Bubble towers for supercritical semilinear elliptic equations. J. Funct. Anal. 221 (2005), no. 2, 251-302.

[16] Ge, Y.; Musso, M.; Pistoia, A. Sign changing tower of bubbles for an elliptic problem at the critical exponent in pierced non-symmetric domains. Comm. Partial Differential Equations 35 (2010), no. 8, 1419-1457.

[17] Gladiali, F.; Grossi, M.; On the spectrum of a nonlinear planar problem. Ann. IHP Anal. Non Lineaire 26 (2009), 191-222.

[18] Grossi, M.; Grumiau, C.; Pacella, A. Lane-Emden problems with large exponents and singular liouville equations (2012) (preprint)

[19] Jost, J.; Wang, G.; Ye, D.; Zhou, C.The blowup analysis of solutions to the elliptic sinh-Gordon equation. Calc. Var. Partial Diff. Equ. 31 (2008) no.2, 263-276.

[20] Li, Y.Y.; Shafrir, I. Blow-up analysis for solutions of $-\Delta u=V e^{u}$ in dimension two. Indiana Univ. Math. J. 43 1255-1270 (1994)

[21] Marchioro, C.; Pulvirenti, M. Mathematical Theory of Incompressible Nonviscous Fluids. Springer, New York (1994)

[22] Moser, J. A sharp form of an inequality by N.Trudinger. Indiana Univ. Math. J. 20 (1970/71), 10771092.

[23] Musso, M.; Pistoia, A. Tower of bubbles for almost critical problems in general domains. J. Math. Pures Appl. (9) 93 (2010), no. 1, 1-40.

[24] Musso, M.; Pistoia, A. Sign changing solutions to a nonlinear elliptic problem involving the critical Sobolev exponent in pierced domains. J. Math. Pures Appl. (9) 86 (2006), no. 6, 510-528.

[25] Ohtsuka, H.; Suzuki, T. Mean field equation for the equilibrium turbulence and a related functional inequality. Adv. Differential Equations 11 (2006), no. 3, 281-304.

[26] Pistoia, A.; Weth, T. Sign changing bubble tower solutions in a slightly subcritical semilinear Dirichlet problem. Ann. Inst. H. Poincaré Anal. Non Linéaire 24 (2007), no. 2, 325-340.

[27] Prajapat, J.; Tarantello, G. On a class of elliptic problem in $\mathbb{R}^{2}$ : Symmetry and uniqueness results. Proc. Roy. Soc. Edinburgh Sect. A 131 (2001), 967-985.

[28] Spruck, J. The elliptic sinh Gordon equation and the construction of toroidal soap bubbles. Calculus of variations and partial differential equations (Trento, 1986), 275-301, Lecture Notes in Math., 1340, Springer, Berlin, 1988.

[29] Trudinger, N.S. On imbeddings into Orlicz spaces and some applications. J. Math. Mech. 17 (1967), 473-483.

[30] Wente, H. Large solutions of the volume constrained Plateau problem. Arch. Rat.Mech. Anal. 75 (1980) $59-77$

[31] Wente, H. Counterexample to a conjecture of H. Hopf. Pacific J. Math. 121 (1986) 193-243

(Massimo Grossi) Dipartimento di Matematica "G.Castelnuovo", Università di Roma "La Sapienza",

P.le Aldo Moro 5, 00185 Roma, Italy

E-mail address: grossi@mat.uniroma1.it

(Angela Pistoia) Dipartimento SBAi, Università di Roma "La SAPienza", via Antonio Scarpa 16, 00161 RomA, ItAly

E-mail address: pistoia@dmmm.uniroma1.it 